\title{
Virtual Reality and Assistive Technologies: A Survey
}

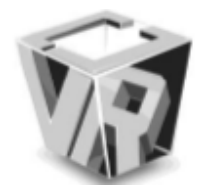

\author{
Shamima Yasmin ${ }^{1}$ \\ ${ }^{1}$ Department of Computer Science, Eastern Washington University *
}

Abstract - This paper conducts an extensive survey on existing Virtual Reality (VR)-based rehabilitation approaches in the context of different types of impairments: mobility, cognitive, and visual. Some VR-based assistive technologies involve repetitions of body movements, some require persistent mental exercise, while some work as sensory substitution systems. A multimodal VR-based environment can incorporate a number of senses, (i.e., visual, auditory, or haptic) into the system and can be an immense source of motivation and engagement in comparison with traditional rehabilitation therapy. This survey categorizes virtual environments on the basis of different available modalities. Each category is again subcategorized by the types of impairments while introducing available devices and interfaces. Before concluding the survey, the paper also briefly focuses on some issues with existing VR-based approaches that need to be optimized to exploit the utmost benefit of virtual environment-based rehabilitation systems.

Index Terms - virtual rehabilitation; mobility impairment; cognitive impairment; visual impairment; assistive technology; haptic.

\section{INTRODUCTION}

A virtual environment (VE) gives the illusion of being there to the user by evoking the sense of presence (Meehan et al. 2002). A VE can be created using a desktop, a frontmounted projection screen with a display or a projected computer display across up to six walls of a room, including floor and ceiling. Users can have the system respond to their actions via different interfaces like a mouse, joystick, Wiimote, and more. Update of a VE can also be associated with realistic controls such as users body movement and walking speed. With stereoscopic vision and a wide field-of-view, users feel themselves immersed in the VE. Integration of force feedback haptic devices can make virtual objects tangible to the users; users can interact with the virtual objects via grasping and squeezing activities while force feedback is felt by them (Popescu, Burdea, and Bouzit 1999). This immersive nature of VEs along with the inherent proprioceptive cues of human beings create illusions to users as though they are in a VE other than where they really are. Thus a

\footnotetext{
* E-mail: syasmin@ewu.edu
}

VE can be an effective source of motivation, enjoyment, and playfulness.

A VE can offer a unique, interactive, and customizable platform that would otherwise be too expensive or not easily implementable in the real world (for example, exploring a fantasy castle, playing with dolphins, and more). A VE is not subjected to the dangers and limitations of the real world. Applications have been developed where users were able to swim with sharks in a VE (Kizony, Katz, and Weiss 2003). A VE can offer an unlimited set of versatility to keep the interests awake. As navigation in a VE is very often associated with different kinds of games, entertainment, or similar activities that require a limited amount of physical exercise or mental awareness or both, a VE can easily be tailored to the needs of different rehabilitation issues specific to different patients. For example, a stroke can cause brain tissue damage and motor loss resulting in partial paralysis. Restoring sensation and movement to the paralyzed limb requires development of new neural pathways through functional reorganization of the surviving motor neurons (Selzer et al. 2006). Unless the patient is actively practicing the movement of a paretic limb, the brain will not undergo substantial plasticity to fully activate the new neural pathways as a substitute to the damaged one (Council 2000). Research results demonstrate that an early and intensive practice produces better results and faster return to normal life (Cameiro et al. 2010). A VE can provide a better sense of engagement (Grealy, Johnson, and Rushton 1999). Users can be forgetful of fatigue and be induced to perform exercise for a longer period of time. VR-based rehabilitation exercises work as an enhanced brain-machine interface to connect brain tissue to task.

A VE is reusable an infinite number of times. Training duration and intensity can easily be manipulated as per the users requirements to meet a certain goal. This can provide the user with immediate visual feedback about their gait and deviations from correct posture by incorporating a self-avatar of the patient and the trainer avatar at the same time (Chua et al. 2003). This can offer better judgment of progress and lesser use of manpower. Physical therapists are also able to create or customize games for use by individual patients. Thus a VE can provide a better scope of intervention.

Very often mobility impairments are found to have a negative impact on patients mental health, such as, depression, attention deficit, forgetfulness, and more. As phys- 
ical health improves, cognitive empowerment takes place. A VE has been used for cognitive rehabilitation of autistic children (Parsons, Leonard, and Mitchell 2006; Herrera et al. 2008). A VE is safe for the treatment of different kinds of phobias or hallucinations (Tarr and Warren 2002; Suárez, Santamara, and Claudio 2013; Anderson et al. 2005). A haptic VE can lead the blind individuals to better accessibility, exploration, and understanding of surrounding objects and environments (Yu, Ramloll, and Brewster 1999; Lecuyer et al. 2003; Moustakas et al. 2007; Lahav and Mioduser 2000; Yasmin and Panchanathan 2016).

As discussed above, VR-based rehabilitation systems can address different types of disabilities. Section 2 briefly touches on the target groups and classifies VEs on the basis of different available modalities. Section 3 to Section 5 survey and analyze different VR-based rehabilitation approaches as developed around different types of VEs as per the classification in Section 2. In a VE, several factors can negatively affect users' gait, physiological response, and presence. Thus Section 6 discusses some drawbacks of VEs. Section 7 summarizes the different approaches to VR-based rehabilitation along with the existing drawbacks while Section 8 concludes the survey.

\section{VIRTUAL ENVIRONMENT AND TARGET GROUPS}

Any disability may be associated with one or more of the following impairments: (a) mobility impairment, (b) cognitive impairment, and (c) visual impairment. Below is a brief description of different kinds of impairments followed by the classification of a VE on the basis of how it addresses different impairments.

Mobility impairments can happen due to several reasons. Patients who have undergone stroke or spinal cord injury, elderly people or Parkinsons patients suffer from motor control deficits that can lead to mobility impairments. Motor paralysis can also be due to traffic accidents, multiple sclerosis (MS), cerebral palsy, neurofibromatosis, traumatic brain injury (TBI), and post-polio syndrome (Adamovich et al. 2009). Depending on the severity of brain tissue damage, movements of the affected limbs can be restricted. In motor paralysis among post-stroke patients, the hand tends to be the most affected limb because of the fact that a large portion of the sensorimotor cortex is allocated to the human hand (Balasubramanian, Klein, and Burdet 2010). Patients grasping and reaching activities can be affected. Patients may even fail to move the paretic arm. When lower limbs are affected, patients may exhibit motor deficits in the form of postural imbalance and longer gait cycles with decreased cadences. Patients suffering from chronic knee injury may also exhibit abnormal gait patterns. Parkinsons patients frequently exhibit freezing gait and persistently struggle to regain normal gait. Patients with frozen shoulder (adhesive capsulitis) suffer from a stiff and painful shoulder with a very limited range of shoulder movement. Post-stroke patients suffering from hemiplegia are left with partial paralysis on one side of the body and movement can be severely restricted in the affected sides hand, arm or leg. People suf- fering from quadriplegia are paralyzed in both arms and both legs and are only capable of moving their faces.

Next comes psychological or cognitive impairment which includes attention or memory deficit, phobia or irrational fear of or aversion to something, hallucinations, or more. Children suffering from neurological disorders like cerebral palsy, autism, elderly people or people undergoing post TBIphase or similar events suffer from functional deficiencies such as little or no response to surrounding events, longer reaction time, mental distraction, forgetfulness, depression, or lack of problem-solving ability. They can have difficulty even in performing the basic activities of daily living (Christiansen et al. 1998). Post-TBI and post-stroke patients may exhibit both cognitive and physical disorders. Symptoms include gaze instability, vertigo, attention deficits, and motion sensitivity as well as clumsy body movement (Rábago and Wilken 2011).

The visually impaired are the next target group. According to WHO classification, this group includes the blind (people with complete or nearly complete loss of vision), legally blind (people with a level of visual impairment considered by law as disabled), or people having a decreased ability to see to a degree that causes problems not fixable by usual means, such as glasses.

In most rehabilitation systems, visual feedback-based virtual environment is used. With the integration of haptic force feedback devices, users can touch, feel or grasp virtual objects. Commonly used exercise equipment such as treadmills or bicycles can also be integrated into a VE to provide the user with better performance metrics. Table 1 categorizes VR-based rehabilitation systems on the basis of subjects requirements and available feedback options (e.g., visual, haptic).

All of the above-mentioned VR-based rehabilitation systems can be enhanced with auditory cues for verbal guidance and environment enrichment. Though in the classification hearing impairment has not been specifically mentioned, all categories of VE can be used for cognitive empowerment of the deaf.

\section{VISUAL FEEDBACK-BASED VIRTUAL ENVIRONMENTS}

Visual feedback-based virtual rehabilitation systems mainly address mobility and cognitive impairments and provide the opportunity to practice under a variety of simulated circumstances. Subjects have to perform different functional tasks with the assistance of a number of devices. Some tasks emphasize body movement while some focus on mental awareness. Table 2 summarizes the classification of visual feedback-based VEs for different rehabilitation approaches using different devices while Figure 1 displays some of these devices.

Among different devices, camera is widely used for tracking and evaluating users movements for interaction purposes. The Wiimote (or Wii remote) developed by Nintendo Co., Ltd. allows the user to interact with and manipulate items in the VE via gesture recognition and pointing through the use of accelerometer and optical sensor technology (Fig- 
ure 1a). The embedded infrared camera in Wii is able to provide kinematic information of the surrounding muscles though a separate camera is required for complex and large movement tracking. It is an input/ output device; some tactile feedback can be generated on collision with virtual objects. For example, users can feel a virtual ball contacting a virtual racket through some haptic sensation which can be enhanced via auditory feedback. Both Wii and camerabased systems interact in a 2D image-based environment. Data glove or CyberGlove Systems CyberGlove can sense the users hand movement, i.e., 3D position or orientation and is able to interact with a 3D environment. CyberGlove Systems CyberGlove II provides a number of sensors that encompass the entire hand to detect the movements (Figure 1b). It is a light weight device freely worn as a hand glove and provides implicit interacting capability with VEs by displaying the changing positions and orientations of the hand.

Microsoft Kinect is an input only device that includes depth sensor to capture video data in 3D and is widely used for 3D skeletal tracking of joints of the body (Figure 1c). Different motion capture systems (i.e., Vicon, Oxford) are used for more accurate 3D kinematics of the patients body. As shown in Figure 1d, a number of retroreflective markers are strapped around the subjects body and several infrared cameras are used to track the markers to construct the skeletal frame, which is used for updating different body movements.

The rest of Section 3 briefly discusses different types of visual feedback-based VR-based rehabilitation systems. Different VR-based rehabilitation systems for mobility impairments have been explained first followed by cognitive rehabilitation and discussion.

\subsection{Visual-feedback-based VEs for mobility impairments}

As demonstrated in Table 2, visual feedback-based physical rehabilitation is classified into two subcategories: (1) VE without full body skeletal tracking and (2) VE with full body skeletal tracking.

In VE-based rehabilitation systems that do not require full body skeletal tracking, movements are simple; tasks in a virtual environment involve movements of different body parts (i.e., wrist, arm, shoulder, knee, and more) or the whole body. Depending on the devices used as mentioned in Table 2 , the following approaches towards rehabilitation have mainly been adopted under this subcategory: (i) camerabased, (ii) Wii-based and (iii) sensor-based. Different applications on these three approaches are discussed below.

In camera-based applications, the user typically sits or stands in front of a camera and image-processing software is used to recognize arm and hand movements (Kizony, Katz, and Weiss 2003; 2004). The user often wears colored gloves or other markers to enable data acquisition. The Rehabilitation Gaming System (RGS) was developed for arm rehabilitation of post-stroke patients where both of the patients arms were tracked by a camera and mapped onto the movements of two virtual arms on the display (Cameiro et al. 2010). This system is demonstrated in Figure $2 \mathrm{a}$. The game required continuous movement of patients arms in the real world to move the virtual arms to intercept a moving sphere as shown on the screen. As soon as the sphere was intercepted, a new position of the sphere was determined which again needed to be intercepted by the virtual arms. Data gloves were used to measure the finger movements.

In order to intensify the degree of realism, instead of virtual limbs, Gesture Xtreme VR system incorporated patients live in-situ video into the VE (Burke et al. 2009; Kizony, Katz, and Weiss 2004). Patients had to constantly move their hands, body, or feet in the real world to make things work in the VE. Figure $2 b$ demonstrates how an individual with spinal cord injury interacting with the Birds and Balls game environment of Gesture Xtreme VR system by persistently moving his body to touch the moving balls to convert them to doves. Similarly, in Figure 2c, a stroke patient is seen to interact with Soccer game env ironment. Sviestrup et al. used a very similar technique to elicit shoulder movements, i.e., shoulder forward flexion, shoulder abduction and shoulder rotation among patients with chronic frozen shoulder (Sveistrup et al. 2003). Patients were superimposed in a virtual soccer game environment and were required to stop the ball from scoring. Multiple interactions and environments were designed to allow for the adjustment of the intensity and difficulty of the body movement. The same interactive VE had been used for balance rehabilitation of TBI patients. Patients were asked to perform several exercises, i.e., picking up objects from the floor, single versus double limb stance, moving within the base of support, walking, hopping, jumping, and jogging. Performance was measured on several issues such as single limb stance, lateral dodging, and stair descent. The VR-exercise group showed a remarkable change in balance and mobility in comparison with the conventional group.

Burke et al. developed some webcam-based rehab games that involved single or bi-manual hand movements (Burke et al. 2009). In Rabbit Chase, a rabbit moved around from hole to hole and the user had to touch the rabbit with one hand as it would come out of the hole. In Arrow Attack, the user had to touch two arrows in a VE: one pointing to the left and another to the right with correct hands, i.e., with left hand and right hand respectively (Figure $2 \mathrm{~d}$ ). These games could be sped up or slowed down depending on the users performance. The users performance was appreciated by rewarding points after each successful completion and by honoring each try with enthusiastic auditory feedback or partial score.

Playstation EyeToy is a low-cost, video-capture system that enables users to interact with virtual objects displayed on a TV screen via active movements of different body parts, i.e., hands, wrists, shoulders, and feet. In the Playstation game Kung Foo, participants repeatedly had to eliminate successive invading warriors by hitting them with the upper limbs; Goal Attack required movement of the whole body with more active movements of upper limbs; Mr. Chef involved cognitive input to prepare different fast food with limited movements of both hands (Figure 2e). Yavuzer et al. evaluated Playstation EyeToy for the treatment of flexion/ extension of the paretic shoulder, elbow, and wrist as well as abduction of the paretic shoulder of post-stroke patients (Yavuzer et al. 2008). In addition to conventional ther- 
apy, playing EyeToy games 5 days a week, 2 to 5 hour per day for four weeks was found to have a potential impact on the enhancement of upper extremity-related motor functioning among post-stroke patients. The Functional Independence Measure (FIM) instrument was used for the evaluation of upper extremity related motor functioning among post stroke patients. The self-care sub-items of FIM that relate to daily activities like eating, grooming, bathing, dressing upper body, dressing lower body, and toileting were considered for the evaluation. Subjects showed significant improvements in self-care activities. The SIGUS platform is a software environment that worked towards the betterment of the lives of quadriplegics. A training dataset associated with different facial features and head movements was created so that the user could associate different visual signs (facial expressions and movements, for instance), to each piece of a virtual drum. After initial calibration, the virtual drum could be played without any direct contact to a mouse or keyboard, just by reproducing a face in front of a standard webcam (Figure 2f). The software was extended with hand gestures and eye gaze (Pistori 2006).

Similar to camera-based applications as discussed above, Wiimotes are widely used for the rehabilitation of shoulder, forearm, wrist and more (Burke et al. 2009; Decker et al. 2010; Alankus et al. 2010; Deutsch et al. 2008). Decker et al. developed Wiihabilitation system for wrist flexion (moving the palm inward) and extension (moving the hand outward) (Decker et al. 2010). The user had to move a paddle in the VE along a horizontal axis through the flexion and extension of their wrist to keep a ball bouncing on the screen. The range of motion of the wrist was measured by attaching reflective tapes which were detected by the embedded infrared camera in the Wiimote. Subjects were found to have increased the range of motion of the wrist as they repeatedly played the game.

Alankus et al. developed a number of Wii-based rehabilitation games while considering patients ability in different levels of recovery (i.e., available motions, cognitive skills, and performance) (Alankus et al. 2010). In the Helicopter game, as a helicopter kept on flying horizontally in the scene, the player needed to move their hands vertically to let the helicopter collect fuel in the air while avoiding hitting the buildings at the same time. Figure $3 \mathrm{a}$ demonstrates a subject playing the game using a Wii remote strapped to the upper arm to detect shoulder flexion. Some Wii-games incorporated social context along with the cognitive input. Under the Sea is a two-player collaborative game which required two patients to move their hands to control and protect a mother fish and its trailing babies from a hungry predator; one player controlled mother fish and babies to collect and eat ferns; another player prevented a predator fish from reaching them. Proper hand coordination and successful control over the game let the patients earn points in the game. Baseball catch required the player screen out baseballs from other types of balls before a catch. To ensure correct muscle groups to be used for movements, additional Wiimotes were attached to the body parts that were supposed to remain stationary. For example, for a game that required only elbow movement, an additional Wiimote was also attached to the upper arm to detect and cancel out the compensation by the rest of the body (Figure 3b).

For wrist and arm rehabilitation, Burke et al. designed Wii-based Virtual vibraphone (Burke et al. 2009). As the user selected a tune for play, each note of the tune gradually highlighted a bar on the virtual vibraphone to be hit with a virtual mallet. In order to play a note, the user had to move the virtual mallet to place it on the highlighted bar by pointing the Wii-remote followed by a press on the controller button. The users score was determined on the basis of how accurately the tune was played within the allocated time. A single user could play two notes simultaneously by holding two Wii-motes with both hands or multiple users could play the application together in a collaborative environment.

Some Wii-based rehab exercises involve the movements of the whole body. Deutsch et al. conducted Wii-based rehab exercises on a patient suffering from cerebral palsy (Deutsch et al. 2008). The patient was asked to play a number of Wii-games (such as tennis, golf, boxing, and more) for practicing body movement often congruent with real-world motions. For example, the Wiimote was held with one hand for bowling, both hands for baseball, tennis or golf, and for boxing, one hand held the Wii-mote and the other a game controller. After undergoing Wii-rehabilitation therapy for a period of four weeks, the patient was reported to have better cognitive ability, more symmetrical posture, and better walking capability.

Similar to Wiimote, sensor-based exergames are also quite popular for the rehabilitation of hands (Burke et al. 2009). In Catch task developed by Burke et al., the user had to hold a real world basket with both hands while magnetic sensors were either attached to the users hands or the basket to catch falling oranges in a virtual basket (Figure 4 a). Game parameters such as the falling speed of oranges or the locus of oranges could be adjusted as per individual capabilities. In Whack a mouse, a mouse appeared at a random location for a few seconds to be whacked by a virtual hammer that was controlled by a sensor attached to the users hand (Figure 4b). Once the user could successfully adapt to this game, they became ready for the next level. In the next level, a dog randomly appeared on the scene and the user had to avoid hitting the dog while whacking the mouse. Adding different levels of difficulty develops a sense of self-competitiveness among the users which make exercises more challenging and engaging at the same time.

Until now, different VR-based rehabilitation applications that do not require full body skeletal tracking were discussed. However, some rehabilitation approaches are about maintaining proper posture and necessitate full body skeletal tracking for continuous adjustment and improvement. Microsoft Kinect and different motion capture systems are used in such applications.

Microsoft Kinect has been widely used for users skeletal tracking and posture determination. Guo et al. replaced existing key-board based movements of the car in an original game with Microsoft Kinect (Guo and Quarles 2013). The users movement was tracked by Microsoft Kinect. Correct body movement increased the speed of the car, incorrect performance resulted in little or no progress. In time-locked op- 
tion, the user needed to perform a particular cycle in an exercise (i.e., crouching and rising) within limited time, otherwise the game would be locked (5a). Other postures such as half-crouching stability did not have time constraints (Figure $5 \mathrm{~b}$ ). Before the game started, participants had to stand for Kinect camera calibration and data initialization. A Bluetooth physiological sensor called Bioharness BT was used to monitor the heart rate. Experimental results demonstrated that participants were able to perform exercises longer with the game.

Espinoza et al. designed Game Cane - a cane-based interface for 3D games for balance rehabilitation where Microsoft Kinect enabled body motion tracking (Espinoza, Cantu, and Quarles 2013). In Game Cane, cane interaction was mapped to game interaction (Figure 5c). To make the user less dependent on the cane and gradually recover balance, an inverse relation was established between the exerted force on the cane and the progress in game. The Wii-mote facilitated interaction with the game and a force sensitive resistor (FSR) was placed at the bottom of the cane to detect the amount of force being applied on the cane (Figure 5d).

Rector et al. developed an audio/visual feedback based yoga exercise program named Eyes-free yoga for the blind and visually impaired to let them maintain an adequate fitness level (Figure 5e) (Rector, Bennett, and Kientz 2013). Microsoft Kinect was used for skeletal tracking of 20 body joints (Figure 5f). A set of rules for each pose was programmed. The exergame offered verbal adjustments and auditory confirmation to assist in pose improvement. Blind participants overall thoughts on eyes-free yoga were positive. As Kinect skeletal tracking does not adapt well to bent knees, poses needed to be compatible with Kinects skeletal tracking, which required users to be in a standing position.

Though Microsoft Kinect is widely used for simple skeletal tracking of the body, some rehabilitation approaches necessitate a very accurate information about the posture of different joints of the body. This can be a stick figure or a virtual human to provide information about joint angles in the different phases of the gait cycle or a trainer avatar embedded into the trainees avatar to provide information about accurate posture (Figure 6a) (Kiefer, Rhea, and Warren 2013). Motion capture based skeletal tracking along with several retroreflective markers on the users body and several infrared cameras is able to provide a very accurate skeletal information of the subjects body.

Chua et al. developed and evaluated a Tai chi training system which aimed to improve training for a full body motor task in an immersive VE by introducing a trainer avatar along with the subjects avatar (Chua et al. 2003). Vicon optical motion capture system was used to compute the skeletal figure of the trainee student for further analysis of the virtual student and their virtual instructor. In the virtual Tai chi environment, the trainee student found himself paired with his virtual instructor in a calm and peaceful $3 \mathrm{D}$ virtual environment as shown to the right of Figure 6b. As the virtual instructor moved for a Tai chi posture, the student in the real world mimicked the virtual instructor and the virtual student was updated as per the joint data of the real student. The updated data was transmitted via a wireless video trans- mitter which was received by a light weight video receiver strapped to the students belt and displayed on the students HMD glass. In order to measure how closely the subject mimicked the teachers motion, a number of comparisons were made between different skeletal parts of the subject and the teacher. In some layout options, a stick figure of the teacher was superimposed inside the students transparent or wireframe body to help the student understand precise movements of the joints and adjust their movements based on the visual feedback.

In order to elicit a better sense of presence, in addition to a responsive full body avatar of the subject himself, an avatar of the assistive device (such as a cane) carried by the subject in real world was also added to the VE (Guo, Samaraweera, and Quarles 2013). Subjects put on an HMD to navigate in the VE while retroreflective markers were fastened to the subjects body and the cane to be tracked by the motion capture system (Figure 6c). In the VE, subjects with mobility impairments used walkways, stairways, and elevators to move around a fantasy castle with the help of a virtual cane as demonstrated in Figure 6d.

\subsection{Visual-feedback-based VEs for cognitive impairments}

Like physical fitness, cognitive empowerment involves gradual change in mental behavior and adaptation to real world via persistent training over a period of time. Cognitive empowerment mainly addresses applications that involve mental exercise. Physical exercise also contributes to mental soundness by promoting both structural and neurochemical changes in the central nervous system (Spirduso and Asplund 1995).

For cognitive empowerment of post-TBI patients, Christiansen et al. designed a virtual kitchen where patients were required to follow a number of steps in order to prepare a can of soup as displayed in Figure 7a (Christiansen et al. 1998). For example, users had to open the cupboard to access the soup can, move the knob of the stove to heat up the cooking surface, boil water, pour necessary ingredients into the can, and so on. In the VE, users were also provided with a series of visual prompts and cues to assist completion of each task. The virtual reality system was comprised of a PC platform and a head-mounted 3-dimensional display and the user interacted with the environment using either a mouse or joystick. Users actions were recorded for subsequent analysis and assessment and they scored points on successful completion of each task. After undergoing VR training over a certain period of time, patients showed a gradual improvement in behavior and were able to perform similar tasks with enhanced prowess in the real world.

Parsons et al. exposed some students with autism spectrum disorder (ASD) to a virtual caf and virtual bus environment to be able to interpret the scenes meaningfully with the help of a facilitator (Parsons, Leonard, and Mitchell 2006). The goal was to learn how to interact with people with appropriate social responses. In both scenarios, there were a number of levels of difficulty; the user's main aim was to find an appropriate place to sit, for example, sitting by oneself or with a stranger (after asking an appropriate ques- 
tion) (Figure 7b). Scenarios also included queuing appropriately, asking someone to move their bags so that they could sit down, and standing when there were no available seats. Verbal responses, providing both positive and negative feedback about the user's behavior, were pre-programmed into the environment and activated as per the users response. In a Desktop-based environment, user interaction was performed with a traditional mouse. Participants were found to be able to properly handle similar real life situations, i.e., in a caf or on a bus and there was a clear transfer of knowledge from VE to real environment.

Herrara et al. let children with ASD shop in a touch screen-based virtual supermarket following a list (Herrera et al. 2008). The goal was to let the children identify different objects and understand their functionalities and social context followed by imaginary transformation of the objects. For example, a pair of trousers were bought to dress up, but were also imagined as a road divided into two. An increased level of attention was observed among the participants on the retest compared to the initial test. The participants understanding of the functional use of each object in the supermarket helped them to use similar objects in a proper way in real life. Participants were also found to exhibit an increase in their ability to distinguish between imaginary and real objects.

Rizzo et al. proposed an intelligent and highly interactive virtual human support agent in their SimCoach project to serve as an online healthcare guide or coach to military service members, veterans for promoting access to psychological healthcare information (Rizzo et al. 2010; 2011). As these people return home from the battlefield, the horror of war keeps on haunting them; they feel stigma and insecurity regarding a clinical condition and tend to live an isolated life. A virtual human coach named SimCoach was designed to be able to engage in rich conversation (even able to recognize nonverbal cues of the user) and provide support and encouragement by increasing awareness, and if necessary, refer to medical practitioners for further diagnosis and treatment. Users would just need sign up to a web-based portal to have access to all services provided by SimCoach. Once logged in, users would be directed to virtual experts on specific areas such as stress, brain injury, marriage counseling, suicide, and so on. SimCoach would store user-specific information and be able to follow up on subsequent visits.

Another application of VE as a cognitive therapy is phobia or hallucination desensitization (Tarr and Warren 2002). This involves gradual exposure of the phobic individuals to circumstances that are increasingly close to the one they are scared of. A VE helps the clinician to measure patients stress level by monitoring their heartbeat and adjust the level of therapy accordingly by modifying it. Surez et al. developed a VR-based training as an effective psychotherapy to gradually shed off excessive fear of height. In the virtual training environment, subjects were taken by an elevator from the ground floor to the topmost floor of a high storey glass building to have a panoramic view of the surrounding landscape (Suárez, Santamara, and Claudio 2013). Similarly, it is easy to simulate a VE with phobiainducing stimuli such as cockroaches, spiders, and more and let the phobic patients confront them in VE so that they can gradually adapt to a similar real world environment. A VE had been recommended for the treatment of schizophrenic patients (Tarr and Warren 2002). Those patients can be infused with virtual hallucinations so that their actual hallucinations can be gradually desensitized. A VE had also been successfully used for the treatment of social phobias such as fear of public speaking by letting people openly address a huge virtual crowd (Anderson et al. 2005; Pertaub, Slater, and Barker 2002; Klinger 2005), for anger management by creating a hostile VE (Rizzo et al. 2003; Macedonio et al. 2007), and for the study of social psychology i.e., racial bias by creating similar virtual scenarios (Blascovich et al. 2002; McCall et al. 2009; Bailenson and Beall 2006).

\subsection{Summary and analysis of different visual feedback-based VEs}

As observed from the above discussion, the treatment goals of visual feedback-based virtual rehabilitation systems are both motor and cognitive empowerment while engaged in some meaningful activity. Research results demonstrate a gradual increase in performance among the subjects. Camera, Wiimote, data glove, or sensors-based VR technologies are relatively cheap, portable and work as a powerful physical and mental therapy. These VR technologies are simple and can be easily set up in the home for practice purposes. When intensive physical therapy is required, the scope of receiving credit (i.e., points in a game or $\mathrm{ku}-$ dos) for successful completion of a virtual task works as an incentive. A multi-player VR game makes the overall training environment social and enjoyable. A visual VE can work as a virtual instructor to guide the subjects for continuous alignment with the proper posture in physical training. With audio integration, a visual feedback-based VE can be catered to the needs of the blind to achieve proper posture in yoga or other popular physical activities. A virtual coach serves as an online healthcare guide or coach for promoting access to psychological healthcare information when subjects may hesitate to break the silence as they become overwhelmed with social stigmas or rituals (Rizzo et al. 2010; 2011). A visual VE has also been successfully used for the treatment of other psychological disorders.

\section{HAPTIC VIRTUAL ENVIRONMENTS}

Haptic relates to the perception using the sense of touch. Perception can be tactile or kinesthetic/ proprioceptive (Fritz and Barner 1999). Tactile perception generates some kind of sensation to the skin; it is unidirectional. Kinesthetic /proprioceptive perception is bi-directional; users move their hands to reach for objects and resistive force is generated to the hand as the object is touched or grasped. Thus kinesthesis is the human sense of position and movement, which is created from proprioceptive cues arising from receptors in the joints and muscles (?; ?). A force feedback based haptic VE can extend a patients muscle capabilities by following the movement of the limb and producing external forces 
proportional to the forces applied by them (Jack et al. 2001; Bouzit et al. 2002). To the blind and visually impaired, touch works as a substitute for vision. A haptic VE has also been successfully used to address attention deficiencies (Reiner et al. 2004; Dvorkin et al. 2009).

Force feedback-based haptic devices can be classified into the following two categories: (a) end effector and (b) exoskeleton. End effectors are non-exoskeletal devices. Some popular end effectors are 3D Systems Touch or Touch X, Novint Technologies Novint Falcon and more. On the other hand, exoskeleton haptic devices provide structural support to the patients paretic limb to further assist in its movement. Among the exoskeleton devices, Rutgers Master II-ND (RMII), CyberGrasp and Haptic Interaction Robot (HIRO) are widely used. Table 3 summarizes the classification of haptic VEs for different types of rehabilitation using different haptic devices.

Among different haptic devices, end effectors are grounded and free standing; users commonly interact with VEs by gripping the handle of the device attached to the end of the device. Figure $8 \mathrm{a}$ and Figure $8 \mathrm{~b}$ demonstrate 3D Systems two such end effectors known as Touch and Touch $\mathrm{X}$ respectively which are widely used for the rehabilitation of wrist and upper limbs. These are 3 degrees of freedom (DOF) force feedback haptic devices with 3D touch workspace and are capable of exerting force up to 3.3 Newton along X, Y and Z directions. 3D Systems Phantom Premium 6 DOF haptic devices are able to produce a larger amount of force and have a much larger work space than Touch devices to consider the movement of the whole arm. In addition to exerting force along $\mathrm{X}, \mathrm{Y}$ and $\mathrm{Z}$ directions, 6 DOF devices are able to apply torque around $\mathrm{X}, \mathrm{Y}$ and $\mathrm{Z}$ axes. The Novint Falcon is a 3 DOF haptic device that was originally designed for the gaming industry by Novint Technologies (Figure 8c). The arm of this device can extend, retract, and fold to measure range of motion. Mirror-image motion enabler (MIME) and HapticMaster are two widely used end effectors specifically designed for motor rehabilitation. MIME can work in bilateral mode, i.e., mirror image movements of the non-affected arm are sent to the robot to guide the movements of the paretic arm (Lum et al. 2005; Loureiro et al. 2011; Tonelo 2013; Wagner 2014).

In comparison with the end effectors, exoskeleton devices can be (i) ungrounded or (ii) grounded. Ungrounded exoskeletal devices are not free standing. Rutgers Master II-ND (RMII) as shown in Figure 9a is a light weight exoskeletal ungrounded robotic device and is used in conjunction with CyberGlove to track finger movements and provide force feedback to the fingers and hand (Popescu, Burdea, and Bouzit 1999; Jack et al. 2001; Bouzit et al. 2002). The force feedback on fingers is provided by the attached actuators to facilitate grasping or other activities in VEs. CyberGlove Systems CyberGrasp as shown in Figure 9b is another ungrounded exoskeletal device that works in a very similar way as RMII, but a bit heavier. The major structural difference between RMII and CyberGrasp is the placement of actuators. In a CyberGrasp system, actuators are placed on the dorsal side while actuators are placed inside the palm in RMII preventing full closure of the hand.
Grounded exoskeleton devices are large and stationary and can be associated with a large and varied amount of force. These devices provide an orthotic shell to support the entire limb. Hand Wrist Assisting Robotic Device (HWARD), ARMin, and MAHI Exo-II are some popular grounded exoskeletons widely used for rehabilitation (Wagner 2014; Nef, Mihelj, and Riener 2007; Guidali et al. 2011; Gupta and OMalley 2007; Sledd and OMalley 2006). HWARD and MAHI Exo-II can work both in active and passive modes. In passive mode, the robot moves the patients arm, unlike in the active assisted mode where the patient moves their arm with the help of the device to reach the desired target.

Haptic Interaction Robot (HIRO) III as shown in Figure $9 \mathrm{c}$ is another grounded multi-fingered exoskeleton haptic interface that provides supports for all fingers to interact with the VE (Endo et al. 2011). HIRO III consists of a 15 DOF haptic hand (3 DOF for each joint in each finger), a 6 DOF interface arm, and a control system. It is placed against a human hand, and the haptic fingertips are connected to the human fingertips through passive spherical magnet joints.

The rest of this section briefly discusses different types of haptics-based VR rehabilitation systems developed with the help of the haptic devices as mentioned in Table 3 for different types of impairments, i.e., mobility, cognitive, and visual.

\subsection{Haptic VEs for mobility and cognitive impairments}

A number of rehabilitation applications have been developed using haptic VEs. Broeren et al. used 3D Systems Touch X device for rehabilitation training of stroke patients with hemiparetic arms (Broeren, Rydmark, and Sunnerhagen 2004). The patient had to grasp the haptic device with their paretic hand to strike a ball to knock down bricks in the VE (Figure 10a). Subjects received credit for knocking down bricks and collected negative points for missing the ball. The game was customized to operate in four different speeds. After playing the game for a period of four weeks in twelve 90-minute sessions, the subject reported that he was able to use his left arm in several Activities of Daily Life (ADL) that were previously impossible for him (e.g., buttoning his shirt, emptying the dishwasher with two hands).

Phantom 1.0, a 3 DOF force feedback haptic device by 3D Systems had been used for VE-based motor rehabilitation training (Jarillo-Silva, Domnguez-Ramrez, and Parra-Vega 2010). Patients had to hold the handle of the device and move their hands along a predetermined trajectory to solve a maze. In active mode, patients guided themselves to follow up the trajectory whereas in passive mode, patients were guided by the device. Experimental results demonstrated that haptic guidance helped patients gradually recover their motor movements.

Dvorkin et al. integrated haptics in VEs to improve attention and concentration in early stages of recovery of the traumatic brain injury (TBI) inpatient population (Dvorkin et al. 2009). It was hypothesized that isolated training with a focused task would be best for restoring attention. Subjects had to interact with the VE by moving the handle of 
Phantom Premium 3.0 (6 DOF) to interact with a number of 3D spheres (Figure 10b). A three-dimensional, largeworkspace haptics/ graphics system called the virtual reality and robotic optical operations machine (VROOM) was used. Integration of augmented reality into the system made the environment more immersive allowing the users to view virtual objects superimposed onto the real world. As the haptic cursor moved towards a sphere, sometimes attractive force was generated; sometimes a repulsive force pushed the hand away from the target sphere. Patients were found to be highly motivated throughout the experiment; the virtual interaction seemed to be well-tolerated, engaging, and enjoyable.

Reiner et al. demonstrated that a visuo-haptic VE creates a better sense of presence among the subjects (Reiner et al. 2004). In this VE, subjects used 3D Systems haptic devices to hit a wall with a number of cubes. While moving the cubes in a VE, some cubes felt heavier than others; some started rolling when touched. Subjects were reportedly found to be highly engaged in the VE.

Ungrounded exoskeleton RMII as demonstrated in Figure 11 had been used for VR-based rehabilitation of stroke patients and orthopedic rehabilitation (Jack et al. 2001). Patients wearing RMII were asked to perform several virtual tasks to determine hand range of motion such as continuously wiping off a window to reveal a different intriguing landscape every time. Some activities required competition with a virtual opponent to measure the speed of movement as the user was asked to catch a virtual ball by closing the palm as fast as possible. In finger fractionation exercises, the movement of an active finger depressed the corresponding key of the virtual piano. RMII had also been used for finger strength exercises that required pressing the attached actuators to fill virtual pistons. Most of the patients showed remarkable improvement in daily functional activities. One post-stroke patient who could not button his shirt was able to do so after he had undergone the training. The same VR system had been used for the treatment of carpal tunnel syndrome that causes numbness and tingling of the thumb, index, middle, and ring fingers. Significant positive effects had been observed among the patients after a number of training sessions (Heuser et al. 2007). The RMII had been used for orthopedic rehabilitation exercises in a VE that involve interactions with dynamic objects and physical modeling of elasticity and plasticity via grasping and squeezing activities while force feedback was felt by the patient (Popescu, Burdea, and Bouzit 1999). Here, instead of a single Haptic Interaction Point (HIP) at each fingertip, a simplified Haptic interaction Mesh (HIM) was considered around each fingertip and the intersection points between the virtual object and HIM were considered in elastic/ plastic deformation simulation.

Grounded exoskeletons like HWARD had been used to practice grasping activities both in real and virtual environments (Wagner 2014). With ARMin, users can practice Activities of Daily Life (ADL) in an enhanced VE with a higher sense of engagement (Nef, Mihelj, and Riener 2007; Guidali et al. 2011). MAHI Exo-II can be used in a virtual reality environment for post-stroke rehabilitation, or for individuals with spinal cord injuries or lacking strength in their arms or wrists (Gupta and OMalley 2007; Sledd and OMalley 2006). HIRO III provides a high precision threedirectional force at the five human fingertips. Figure 12 demonstrates the interaction mechanism with HIRO III and the positions of the fingertips as shown with red dots while grasping, lifting, and moving a virtual object (Endo et al. 2011). Grounded exoskeletons are found to be highly effective in the rehabilitation of upper limb paralysis because they can be applied to multiple joints. This flexibility allows researchers to investigate rehabilitation of fine motor control and a larger range of motion (Wagner 2014).

\subsection{Haptic VEs for visual impairments}

A haptic VE can work as an effective learning platform for the blind and visually impaired. This can also enhance social interaction capabilities of the blind through a better understanding and evaluation of the surroundings (Yasmin and Panchanathan 2016). The conventional method of teaching blind students is through raised line drawings, cutout tiles, or hardboards with complex figures, or cross-sections embossed. VEs proved to be more flexible and scalable. In a VE, a haptic line chart was initially presented by a haptic cylinder or embossed ridge to feel with force feedback haptic devices provided by 3D Systems (Yu, Ramloll, and Brewster 1999; 2000; Roberts, Franklin, and Cullinane 2002). Later, attraction forces were used to keep the user on the line (Panëels et al. 2013). When the Haptic Interaction Point (HIP) was close enough to the line, it was pulled towards the line. Once on the line, no forces were generated to resist the motion of the HIP along the line, but motion away from the line was opposed and a relatively large force was required while attempting to pull away from the line. This helped the blind user to find and trace the line in a tangible line chart. The blind user could haptically explore some points of interest, i.e., maximum, minimum, and inflexion points on the graph. While haptic navigation, non-speech audio demonstrated the overview of the data set by mapping MIDI notes pitch to the $\mathrm{Y}$-axis values while $\mathrm{X}$-axis was mapped to time. Synthesized speech was used to provide the user with exact coordinate values in the chart.

Figure 13 displays a variety of haptic bars, charts, and so on. Yu et al. proposed that engraved lines work better than an embossed ridge as this prevent the user from falling off the surface while navigating in non-constrained mode (Figure 13a) (Yu, Ramloll, and Brewster 2000; Yu and Brewster 2002). Like line graphs, different bars or sections of a pie chart were also explored for haptic data representation as demonstrated by Figure $13 \mathrm{~b}$ and Figure $13 \mathrm{c}$ respectively.

Engraved bars enabled the user to stick to the bar and prevented slip-off (Yu, Ramloll, and Brewster 2000; Yu and Brewster 2002). In haptic bar charts, audio pitch corresponded to the $\mathrm{Y}$ value of the top of the bar. Haptic pie charts were built with resistive walls to form an enclosure for each segment, and a groove around the edge was used to provide an overview of the information (Yu, Cheung, and Brewster 2002). In haptic pie charts, pitch value was made proportional to the length of the arc. Different frictional forces helped to haptically distinguish different lines in a line chart, 
different bars in a bar chart, and sections in a pie chart.

Panels et al.worked on haptic-based representation of scatter plot for the blind (Panëels and Roberts 2009). In a scatter plot, data is displayed as a collection of points to understand the relationship between the variables through the size and location of the point cloud, the direction of the relationship, and whether outliers exist. A haptic scatter plot was modeled by assigning a repulsive force to each point. While exploring the plot, a greater force was felt for larger concentrations of data points (Figure 13d). In a haptic representation of 3D surface plot, polygons or implicit functions were used for collision detection (Fritz and Barner 1999).

In a haptic-based representation, variation in data was also represented via different surface properties, i.e., surface friction, stiffness, texture, viscosity, and so on (Wu, Song, and Zou 2007). The higher the surface friction, the higher resistive force is felt by the user during haptic navigation. Surface textures are usually generated by extracting data height from image processing, perturbing surface normals, creating noise in the data set, and more (Figure 13e). Haptic texturing was used for a representation of data variables or different regions, in the same fashion as color is used in a vision-based representation (Fritz and Barner 1999).

Van Scoy et al. implemented Haptic math where mathematical functions were represented as virtual grooved curves. Users were able to interact with the system with a haptic device and audio feedback (Van Scoy et al. 2000).

Moustakas et al. introduced a force-field haptic rendering method of 3D maps to let the visually impaired navigate these maps using off-the-shelf haptic devices (Moustakas et al. 2007). Users were found to be enthusiastic about the system and later, asked for options to be able to zoom in on to specific areas of the map for further investigation. Lahav et al. concluded that blind peoples spatial performance can be enhanced if appropriate spatial information is provided through compensatory channels (Lahav and Mioduser 2000). In their experiment, blind individuals were asked to haptically explore a VE that simulated real room with objects. Following the exploration task, they were asked to give a verbal description of the environment and construct a scale model of it. Haptic feedback and haptic exploration enabled and helped the blind individuals develop a highly accurate cognitive map of the environment. Parente et al. proposed Blind Audio Tactile System (BATS) where geographical information were presented with different haptics-based feedback: subtle bumps for country boundaries, large bumps for state boundaries, and a constant vibration on cities (Parente and Bishop 2003). Speech had been used for objects names and descriptions and also for directions. Non-speech auditory icons had been added to provide surrounding information: traffic sounds for cities, bird chirping for forests, and so on (Ko 2000).

Lecuyer et al. designed a haptic and audio-based multimodal application called HOMERE to explore and recognize the environment to prepare the blind for an independent visit to an existing site in a real world (e.g., tourist site, administrative building, airport, or train station) by ludic exploration in the virtual world (Lecuyer et al. 2003). As demonstrated in Figure 14a, the user could detect obsta- cles and feel the textures on the ground or on some objects in the VE with a virtual cane. In a HOMERE system, thermal feedback corresponded to the simulation of a virtual sun while an auditory feedback corresponded to the ambient atmosphere or oral guidance from a virtual avatar. A visual feedback of the scene was also provided for the sighted user. HOMERE has been tested by several visually impaired people. All were found to be enthusiastic and confident about the potential of the developed prototype.

Nikolakis et al. used 3D Systems Touch X haptic device in combination with Cybergrasp and Cyberglove to develop a customized VR set up with full five-finger haptic feedback (Nikolakis et al. 2004). The haptic device allowed the visually impaired to explore details of the object, while the CyberGrasp helped the user perceive the general shape and size of the object (Figure 14b). The single interaction point of the haptic device provided force feedback onto the index finger. With the help of CyberGlove, the positions of all finger tips were determined and the positional data of the fingertips were used for collision detection between the fingers and the virtual object. Next, output force for all finger tips were generated. In the application, an audio- haptic menu assisted the user to select an object. The selected object declaimed itself via audio feedback. Next, the user could explore the object haptically while listening to information about it.

Yasmin et al. developed a haptic mirror to enrich the abilities, lives, and self-sufficiency of blind individuals in interpreting different facial expressions and assist in understanding non-verbal cues of interaction partners in social communication (Yasmin and Panchanathan 2016). It was hypothesized that persistent haptic exploration of different movements of ones own facial features for different expressions in the VE would help a blind individual master the haptic language of facial expressions. Now, when others facial data would be accessed through the VE, the blind would be able to interpret others facial expressions. At first, facial data were collected via a webcam. Next, extracted features were normalized, enhanced, and haptically augmented. Figure $14 \mathrm{c}$ demonstrates the proof-of-concept Haptic mirror prototype in use by a blind participant. The preliminary findings convincingly supported the idea and social relevance of the described system, its necessity, and acceptability by the target users.

\subsection{Summary and analysis of haptic VEs}

As discussed above, a haptic VE enhances mobility and cognitive impairments and works towards the enrichment of the lives of the blind and visually impaired. Patients finger dexterity, grip force, endurance, and attention were found to have improved significantly after undergoing training with the haptic devices (Broeren, Rydmark, and Sunnerhagen 2004). In motor-rehabilitation training, force feedbackbased VR therapy was found to be more effective and produced faster results in comparison with visual feedback-only VR systems (Jack et al. 2001). Grounded exoskeletons with their large workspace, ability to work in different modes (i.e., from initial passive mode to active assisted mode to fully active mode) and orthotic shells ensure gradual mo- 
tor recovery of an entire limb in a safe and engaging environment. A haptic VE improves the accessibility of educational materials for blind students and helps them mentally form an accurate representation of their surroundings (Gandhi, Ganesh, and Sinha 2014). Onset blindness produces alterations to spontaneous facial behavior and is found to be associated with symptoms like more closed eyes, open mouth, raised eyebrows, and more (Matsumoto and Willingham 2009). Hence children with visual disabilities find it very difficult to learn social skills while growing amongst sighted peers (Krishna et al. 2010). If applications like the haptic mirrors can be incorporated as a learning tool, as these children grow up, they can have a better understanding of the real world and be better able to communicate with their surroundings (Yasmin and Panchanathan 2016).

\section{VIRTUAL ENVIRONMENTS ENHANCED WITH EXERCISE EQUIPMENT}

A VE can be enhanced with exercise equipment to improve postural imbalance. Visual scenes of a corridor / landscape are projected onto a large screen. The visual update is synchronized with the walking speed on a treadmill or the speed of a bicycle. Table 4 summarizes VEs enhanced with exercise equipment to be used as a physical therapy.

The rest of Section 5 discusses different VR-based rehabilitation systems which evolved around different exercise equipment, i.e., treadmill, bicycle, and more.

\subsection{VR-enhanced treadmill}

In VR-enhanced treadmills, VR environment, field of view, instrumental set-ups as well as the treadmill walking surface differ from system to system (Sheik-Nainar and Kaber 2007; Fung et al. 2006; Gates et al. 2012; Sloot, van der Krogt, and Harlaar 2014a; van der Krogt et al. 2015; Hollman et al. 2006; 2007; Katsavelis et al. 2010; Sloot, van der Krogt, and Harlaar 2014b; Tierney et al. 2007). The Computer-Assisted Rehabilitation Environment (CAREN) treadmill system is comprised of a 7-m diameter dome with 300-degrees of visual field projection (Gates et al. 2012). In some VR-enhanced systems, a three dimensional landscape of a corridor or hallway is projected onto a semicylindrical or concave screen in front of the treadmill (Sloot, van der Krogt, and Harlaar 2014a; van der Krogt et al. 2015; Hollman et al. 2006; 2007). Additional ground projection of the scene adds better immersion. This technique creates a wide field of view and subjects do not need to wear stereo glasses or head mounted devices. Some other systems project a 3D scene onto a flat screen in front of the subjects resulting in a much smaller field of view (50 to 60 degrees) (Sheik-Nainar and Kaber 2007; Katsavelis et al. 2010). Subjects have to wear stereo glasses or HMD for immersive effect. In CAREN systems, subjects walk on a singlebelt treadmill embedded in a motion platform (Gates et al. 2012). Gait Real-time Analysis Interactive Lab (GRAIL) as demonstrated in Figure 15a used an instrumented splitbelt treadmill (Sloot, van der Krogt, and Harlaar 2014a; van der Krogt et al. 2015). A fixed-paced treadmill allows subjects to maintain a constant walking speed and is recommended in their early stage of rehabilitation. Visual scenes are updated at a constant speed as maintained by the subject. On a self-paced treadmill, the speed of the belt is real time adjusted to the subjects time-varying walking speed; optical flow is continuously matched to the walking speed of the subject (Sloot, van der Krogt, and Harlaar 2014a; 2014b).

In the CAREN treadmill system, 3D kinematics of the lower body are tracked using motion capture system (i.e., Vicon, Oxford, UK) and used to calculate angular motion of joints, i.e., ankle, knee and hip. People with mobility impairments typically present abnormal patterns of joint moments and powers during gait. For proper gait rehabilitation, it becomes necessary to compute kinetic information i.e., the minimum force level that muscles need to produce at any instant during the gait cycle. In Gait Real-time Analysis Interactive Lab (GRAIL), force sensors underneath each belt of an instrumented split-belt treadmill records the ground reaction forces (GRF) and moments (Sloot, van der Krogt, and Harlaar 2014a; van der Krogt et al. 2015). Force and motion data are used to assess hip, knee, and ankle joint moments and powers as well as the center of pressure (CRP).

The CAREN treadmill system requires subjects to put on a harness attached to a support structure to ensure an increased feeling of safety (Gates et al. 2012). Tierney et al. worked towards developing a VR-enhanced treadmill called VR-gait system as shown in Figure $15 \mathrm{~b}$ that provided patients with adjustable body weight support (Tierney et al. 2007). As the subjects walked through the VE displayed on a high resolution monitor mounted in front of the treadmill, an inertial orientation tracking device determined whether the subjects maintained correct posture, i.e., looking up or down or leaning to one side. An instant auditory feedback via a friendly virtual avatar was delivered to the subjects. The difficulty levels of the training scenarios were adjusted by varying the speed and slope of the treadmill, the complexity of tasks, and the amount of body weight support.

\subsection{VR-enhanced bike system}

Kim et al. developed and assessed a VR-enhanced bike system for the rehabilitation of postural imbalance. The system consisted of two parts: one bike simulator software that generated virtual space and a sensing part attached to an in situ bicycle (Kim, Song, and Kim 2001). As the subject pedaled through the VE, the head movement was tracked; the orientation angle of the handle bar and driving velocity were measured (Figure 16a). A force plate at the bottom of the cycle was used for the measurement of the subjects center of pressure (COP) and the subjects weight shift was measured from the left and right body weight distribution. The experimental results showed that after virtual cycle training, subjects could more easily control the handle bar and there was a reduction in path deviation with a balanced distribution of body weight.

\subsection{VR-enhanced leg orthosis driven treadmill}

Wellner et al. used a VR-enhanced gait rehabilitation robot named Locomat to automate treadmill training of patients 
with spinal cord injury or stroke [92]. The robot was driven by the weight and friction of the leg orthosis worn by the subject as shown in Figure 16b. The force sensors embedded at the bottom of the treadmill were used to measure the shear force between the feet and the treadmill. The resulting force was used to accelerate the treadmill. The obstacles in the VE generated haptic force feedback to the subject. This was computed from the penetration depth of the foot tip into the obstacle which resulted in a force driving the foot out of the obstacle. Audio feedback consisted of event-driven sounds (i.e., steps, obstacle hits, and background music). Based on the knee and hip angles of the orthosis, a virtual avatar of the patient walking over the obstacles was also displayed as demonstrated in Figure 16b.

\subsection{Summary and Analysis of VEs enhanced with exercise equipmentl}

Stroke patients exhibited improved walking speed and posture in a VR-enhanced treadmill training system in comparison with training systems that did not incorporate VEs [82]. Gates et al. successfully deployed a VR-enhanced treadmill training system for the gait rehabilitation of patients with transtibial amputation [83].The scope of continuous visual representation and analysis of gait using motion capture systems help trainees constantly maintain proper gait while walking on a treadmill. Research findings also demonstrated that visual stimulus of continuous visual feedback was more effective than that of nonvisual feedback in the postural balance control (Wellner et al. 2007). Incorporating haptic feedback in VE and synchronizing it with the users movement on a treadmill can make the overall training environment more interesting and engaging. The scope of measurements of both kinetic and kinematic metrics of a subjects postural balance makes the findings ready for further clinical diagnosis. Adjusting task complexity according to the level of performance makes the overall training environment flexible and challenging at the same time.

\section{DRAWBACKS IN VR-BASED REHABILITATION}

A VE can negatively affect users' gait, physiological response, and presence. A delay between a users actions and consequent changes in the virtual world can destroy the sense of engagement and can lead to physical disorientation and nausea (Haffner November 19 1998; Durgin, Gigone, and Scott 2005). The lack of humanness of virtual characters and spontaneity in interaction can distract the level of immersion of the subjects too. Some participants stated that the VE seems unnatural as they found it challenging to coordinate the real movement to a virtual grasp of virtual objects, even with assistance of the devices (Guidali et al. 2011). Additionally, virtual devices like HMDs can be a source of encumbrance that would likely hinder the motor response of patients with neurological deficits. Sometimes users wearing an HMD could see a part of the real world due to a limited vertical field of view and their attention was distracted while walking in a VE [37].
Sometimes subjects reported difficulty in exacting a performance even with a virtual trainer with accurate body movement superimposed on their own virtual avatars (Chua et al. 2003). They also reported to feel more fatigued in a VR-enhanced environment (Espinoza, Cantu, and Quarles 2013). These findings may be attributed to the fact that a virtual body in a VE could increase the presence of focusing on visual senses and users become more conservative and cautious while exacting the performance (Schultze 2010; Slater and Usoh 1993). Sometimes patients were found to be frustrated while playing a particular game if the game does not involve sufficient amount of motivation or engagement. It was also reported that participants seemed to be more interested in visually exploring the environment rather than inspecting the avatars for posture control (Guo, Samaraweera, and Quarles 2013). In some experimental results, the percentage of time in good form was found to be lower in games compared with the real world (Guo and Quarles 2013). Thus visual cues and avatars can negatively affect gait in velocity, stride length, and step length.

Several studies resulted in contradictory remarks as addition of a VR environment does not normalize the comfortable walking speed, but lowers the stride length with increased step width (Hollman et al. 2006; 2007). Mohler et al. found that a person wearing an HMD and backpack walks slower, and takes a shorter stride length in a VE than they do in a comparable real world condition (Mohler et al. 2007). However, Phillips et al. found that a person takes significantly shorter strides in a stressful VE compared to a controlled VE (Phillips et al. 2010). Different evaluation methods regarding users performance improvement like Functional Independence Measure (FIM) focus on the performance of the individual on a specific activity. FIM does not consider whether a particular VE-based rehabilitation has contributed to the development of compensatory self-care strategies by the non-paretic side over time or the paretic limb (Yavuzer et al. 2008). A very few research works were found to consider this issue in performance evaluation (Decker et al. 2010).

Parsons et al. commented that some autistic children failed to demonstrate social use of the lessons learnt in a VE; participants learn how to use VE but not where to use it (Parsons, Leonard, and Mitchell 2006). The reason was mainly attributed to the paucity of social contact available to these participants. For better outcome, not only the cognitive play in VE but also broader exposure to social environments and wider social interaction would be necessary.

Different VR-enhanced systems can affect subjects in different ways. Participants in the low latency condition had a higher self-reported sense of presence (Meehan et al. 2002). Though participants were found to walk more slowly in VEs, they commented that the avatar made walking in the VE more realistic (Samaraweera, Guo, and Quarles 2013). Research must also take into account individual differences because each persons ability to perform a particular task varies from others. VEs, games, or devices that work for some individuals might not work for others. This variance can greatly impact the level of improvement of each person undergoing rehabilitation. Thus a more customized device or 
virtual task help the participants interact better (Broeren, Rydmark, and Sunnerhagen 2004).

\section{DISCUSSIONS ON VR-BASED REHABILITATION}

In a VR-based rehabilitation, a target-oriented training along with a suitable VR platform is essential for faster recovery. For example, with HMD, certain participants reported to experience dizziness or nausea when exposed to a VE for a long period of time (Kizony, Katz, and Weiss 2004). Though HMD provides better immersion, these participants should avoid using HMD or options can be provided for switching between monitor and HMD. Though latency is an issue with VR, all VR-based applications do not suffer from it; choosing a suitable VR platform along with a suitable virtual task can minimize this problem.

Most often motor recovery is associated with intensive therapy that requires the user to perform hundreds of daily repetitions of motions of the paretic limb. But only one third of post-stroke patients were reported to perform exercises as recommended by their therapists (Alankus et al. 2010). Traditional one-on-one therapy is labor-intensive and expensive. As discussed above, a VE provides continuous and gradual adaptation of the exercise parameters to the users performance and ability under a variety of simulated circumstances. Wii-motes or other visual feedback-based assistive technologies are portable, affordable, flexible, and good for practice at home for necessary motor recovery. These assistive technologies are simple, yet powerful in rehabilitation. Integarting popular exercises like crouching or halfcrouching along with progress in a game makes the overall environment less monotonous. A VE enhanced with exercise equipment works as a better practice ground than nonVR systems for continual development of kinesthetic memory via intensive training of the paretic limb.

In motor-rehabilitation training, force feedback haptic devices not only address patients kinematics but also focus on the kinetics of the hand and wrist by assisting patients grasp of virtual objects with their deficient arm. Thus force feedback-based VR therapy was found to be more effective and produced faster results in comparison with visual feedback-only VR systems (Jack et al. 2001). This haptic feedback can be adapted to specific tasks to be fully exploitable (Wagner 2014). The device handle can be replaced to incorporate different removable grips, i.e., thumbpad, thimble, or hand glove to facilitate interaction with the VE.

A force feedback-based dynamic VE could prove to be more flexible and scalable, giving eventually access to unlimited sets of educational cases to the blind in comparison with conventional method of teaching. Though in our discussion, it has not been explicitly mentioned about the empowerment of the deaf, all VR-based rehabilitation systems can be deployed for the cognitive empowerment of the deaf individuals and be quite helpful for better integration of such population into the mainstream of society As shown in Figure 17a, using a conventional Tadoma technique, a deafblind individual physically accesses others mouth to receive a message. People who are both deaf and blind find difficulty while communicating among themselves especially when communication involves more than two people. Applications such as a haptic mirror may facilitate communication among deafblind individuals as well; while one person wants to communicate, others will be able to examine his haptic facial features, and understand what message he wants to convey (Yasmin and Panchanathan 2016). In order to facilitate better interaction, dynamic tactile pin arrays may be used so that the blind can feel the VE with their own hands. This scenario is demonstrated in Figure $17 \mathrm{~b}$.

As discussed in the previous section, question arises whether putting on an extraneous outfit like HMD or interacting via haptic devices can persistently affect patients performance and rehabilitation. Again, choosing a suitable VR application can be a solution. As the philosopher Polyani observed that prior to full achievement of a skill (e.g., learning and interacting with a new interface), the users awareness focuses on the components of the skill. As skill develops, the user eventually becomes more focused on the contents of the VE, and less aware of the interface used in it (Loomis 1992). After becoming accustomed to the VE, users eventually experience more natural interaction. Apart from that, performance during practicing may differ not only between the VR platforms but can also be influenced by the gender or age of the participants and the virtual task to be performed (Rand et al. 2006). Male participants may perform better during virtual soccer games while their female counterparts may be more motivated to a virtual fashion show. Thus if negative findings with respect to a VE can be carefully considered, side effects can be substantially reduced.

\section{CONCLUSION}

This paper classifies VR based applications on the basis of their characteristics to assist in choosing, extending, or developing a suitable rehabilitation therapy for the affected group. VR-based physical therapies can be used or extended for cardiac or other rehabilitation; cognitive therapy can be adjusted for drug rehabilitation using suitable context. Intensive training leads to better health benefits and incentive leads to hard work (Whitehead et al. 2010). VR-based rehabilitation systems are capable of providing both incentive and physical benefit to the subjects in a safe and secure environment, which may not be possible with conventional therapy. A VE proves to be more flexible and scalable than conventional rehabilitation systems. Research findings demonstrate that a VE facilitates a consistent transfer of skills from the simulated environment to the real world. VEs have been successfully used in the design and development of different assistive tools. Slight drawbacks in VR-enhanced rehabilitation systems can be easily addressed and outweighed by the gains of using a VE. Research works are being carried out so that latency during interactions with a VE can be optimized. VEs are getting more life-like; while interacting with a VE, subjects were even reported to be forgetful of their real world existence. It was demonstrated that patients improvements, trained in most of the VR contexts, were retained by them outside of the VE. 


\section{REFERENCES}

[1] Adamovich, S. V.; Fluet, G. G.; Tunik, E.; and Merians, A. S. 2009. Sensorimotor training in virtual reality: a review. NeuroRehabilitation 25(1):29-44.

[2] Alankus, G.; Lazar, A.; May, M.; ; and Kelleher, C. 2010. Towards customizable games for stroke rehabilitation. In Proceedings of the SIGCHI Conference on $\mathrm{Hu}$ man Factors in Computing Systems (CHI10), 2113-2122.

[3] Anderson, P. L.; Zimand, E.; Hodges, L. F.; and Rothbaum, B. O. 2005. Cognitive behavioral therapy for public speaking anxiety using virtual reality for exposure. Depression and Anxiety 22(3):156-158.

[4] Bailenson, J. N., and Beall, A. C. 2006. Transformed social interaction: exploring the digital plasticity of avatars. In Avatars at Work and Play: Collaboration and Interaction in Shared Virtual Environments, $R$ Schroeder and A Axelsson, Eds, Springer-Verlag, Berlin, Germany, 1-16.

[5] Balasubramanian, S.; Klein, J.; and Burdet, E. 2010. Robot-assisted rehabilitation of hand function. Current Opinion in Neurology 23(6):661-670.

[6] Blascovich, J.; Loomis, J.; Beall, A.; Swinth, K.; Hoyt, C.; and Bailenson, J. 2002. Immersive virtual environment technology: not just another research tool for social psychology. Psychological Inquiry 13:103-124.

[7] Bouzit, M.; Burdea, G.; Popescu, G.; and Boian, R. 2002. The rutgers master ii-new design forcefeedback glove. IEEE/ASME Transactions on Mechatronics 7(2):256-263.

[8] Broeren, J.; Rydmark, M.; and Sunnerhagen, K. S. 2004. Virtual reality and haptics as a training device for movement rehabilitation after stroke: A single-case study. Archives of Physical Medicine and Rehabilitation 85(8):1247-1250.

[9] Burke, J. W.; McNeill, M.; Charles, D.; Morrow, P.; Crosbie, J.; and McDonough, S. 2009. Serious games for upper limb rehabilitation following stroke. In Proceeding of Games and Virtual Worlds for Serious Applications, 103-110.

[10] Cameiro, S.; Bermdez i Badia, S.; Oller, E. D.; and Verschure, P. 2010. Neurorehabilitation using the virtual reality based rehabilitation gaming system: methodology, design, psychometrics, usability and validation. Journal of neuroengineering and rehabilitation 7(48):1-14.

[11] Christiansen, C.; Abreu, B.; Ottenbacher, K.; Huffman, K.; Masel, B.; and Culpepper, R. 1998. Task performance in virtual environments used for cognitive rehabilitation after traumatic brain injury. Archives of physical medicine and rehabilitation 79(8):888-892.

[12] Chua, P. T.; Crivella, R.; Daly, B.; Hu, N.; Schaaf, R.; Ventura, D.; Camill, T.; Hodgins, J.; and Pausch, R. 2003. Training for physical tasks in virtual environments: Tai chi. In Proceedings of IEEE Virtual Reality 2003 (VRO3), 87-94.
[13] Council, N. R. 2000. How People Learn: Brain, Mind, Experience, and School: Expanded Edition. Washington, D.C.: National Academy Press.

[14] Decker, J.; Li, H.; Losowyj, D.; and Prakash, V. 2010. Wiihabilitation: rehabilitation of wrist flexion and extension using a wii-mote-based game system. In Rutgers University, Governor's School of Engineering and Technology (GSET 09).

[15] Deutsch, J. E.; Borbely, M.; Filler, J.; Huhn, K.; and Guarrera-Bowlby, P. 2008. Use of a low-cost, commercially available gaming console (wii) for rehabilitation of an adolescent with cerebral palsy, physical therapy. Physical Therapy 88(10):1196-1207.

[16] Durgin, F. H.; Gigone, K.; and Scott, R. 2005. Perception of visual speed while moving. Journal Exp Psychological Human 31(2):339353.

[17] Dvorkin, A. Y.; Zollman, F. S.; Beck, K.; Larson, E.; and Patton, J. L. 2009. A virtual environment-based paradigm for improving attention in tbi. In Proceedings of IEEE International Conference on Rehabilitation Robotics (ICORR 2009), 962-965.

[18] Endo, T.; Kawasaki, H.; Mouri, T.; Ishigure, Y.; Shimomura, H.; Matsumura, M.; and Koketsu, K. 2011. Five-fingered haptic interface robot: Hiro iii. IEEE Transactions on Haptics 4(1):14-27.

[19] Espinoza, E.; Cantu, M.; and Quarles, J. 2013. Converting sedentary games to exergames: a case study with a car racing game. In Proceeding of 1st Workshop on Virtual and Augmented Assistive Technology (VAAT13), 23-26.

[20] Fritz, J. P., and Barner, K. E. 1999. Design of a haptic data visualization system for people with visual impairments. IEEE Transactions on Rehabilitation Engineering 7(3):372-384.

[21] Fung, J.; Richards, C. L.; Malouin, F.; McFayden, B. J.; and Lamontagne, A. 2006. A treadmill and motion coupled virtual reality system for gait training poststroke. Cyberpsychology and Behavior 9:157-162.

[22] Gandhi, T. K.; Ganesh, S.; and Sinha, P. 2014. Improvement in spatial imagery following sight onset late in childhood. Psychological Science 25(3):693-701.

[23] Gates, D. H.; Darter, B. J.; Dingwell, J. B.; and Wilken, J. M. 2012. Comparison of walking overground and in a computer assisted rehabilitation environment (caren) in individuals with and without transtibial amputation. Journal of NeuroEngineering and Rehabilitation 9(81):1-10.

[24] Grealy, M. A.; Johnson, D. A.; and Rushton, S. K. 1999. Improving cognitive function after brain injury: the use of exercise and virtual reality. Archives of Physical Medicine and Rehabilitation 80(6):661-667.

[25] Guidali, M.; Duschau-Wicke, A.; Broggi, S.; Klamroth-Marganska, V.; Nef, T.; and Riener, R. 2011. A robotic system to train activities of daily living in a virtual environment. Medical \& Biological Engineering \& Computing 49(10):1213-1223. 
[26] Guo, R., and Quarles, J. 2013. Converting sedentary games to exergames: a case study with a car racing game. In Proceeding of 5th International Conference on Games and Virtual Worlds for Serious Applications (VS-GAMES 2013), 1-8.

[27] Guo, R.; Samaraweera, G.; and Quarles, J. 2013. The effects of ves on mobility impaired users: presence, gait, and physiological response. In Proceedings of the 19th ACM Symposium on Virtual Reality Software and Technology (VRST13), 59-68.

[28] Gupta, A., and OMalley, M. K. 2007. Robotic exoskeletons for upper extremity rehabilitation. In Tech Education and Publishing, Vienna, Austria, 371-396.

[29] Haffner, K. November 19, 1998. Real queasiness in virtual reality. In The New York times.

[30] Herrera, G.; Alcantud, F.; Jordan, R.; Blanquer, A.; Labajo, G.; and DePablo, C. 2008. Development of symbolic play through the use of virtual reality tools in children with autistic spectrum disorders: two case studies. Autism 12(2):143-157.

[31] Heuser, A.; Kourtev, H.; Winter, S.; Fensterheim, D.; Burdea, G.; Hentz, V.; and Forducey, P. 2007. Telerehabilitation using the rutgers master ii glove following carpal tunnel release surgery: proof-of-concept. IEEE Transactions on neural systems and rehabilitation engg 15(1):43-49.

[32] Hollman, J. H.; Brey, R. H.; Robb, R. A.; Bang, T. J.; and Kaufman, K. R. 2006. Spatiotemporal gait deviations in a virtual reality environment. Gait and Posture 23(4):441444.

[33] Hollman, J. H.; Brey, R. H.; Bang, T. J.; and Kaufman, K. R. 2007. Does walking in a virtual environment induce unstable gait? Gait and Posture 26(2):289294.

[34] Jack, D.; Boian, R.; Merians, A. S.; Tremaine, M.; Burdea, G. C.; Adamovich, S. V.; Recce, M.; and Poizner, H. 2001. Virtual reality-enhanced stroke rehabilitation. IEEE Transactions on Neural Systems and Rehabilitation Engg 9(3):308-318.

[35] Jarillo-Silva, A.; Domnguez-Ramrez, O. A.; and ParraVega, V. 2010. Haptic training method for a therapy on upper limb. In Proceedings of 3rd International Conference on Biomedical Engineering and Informatics (BMEI 2010), 1750-1754.

[36] Katsavelis, D.; Mukherjee, M.; Decker, L.; and Stergiou, N. 2010. The effect of virtual reality on gait variability. Nonlinear Dynamics, Psychology, and Life Sciences 14(3):239256.

[37] Kiefer, A. W.; Rhea, C. K.; and Warren, W. H. 2013. Vr-based assessment and rehabilitation of functional mobility. Human walking in virtual environments, Springer, New York 333-350.

[38] Kim, J. Y.; Song, C. G.; and Kim, N. G. 2001. A new vr bike system for balance rehabilitation training. In Proceedings of Seventh International Conference on Virtual Systems and Multimedia, 790-799.
[39] Kizony, R.; Katz, N.; and Weiss, P. L. 2003. Adapting and immersive virtual reality system for rehabilitation. Journal of Visualization and Computer Animation 14(5):261-268.

[40] Kizony, R.; Katz, N.; and Weiss, P. L. 2004. Virtual reality based intervention in rehabilitation: relationship between motor and cognitive abilities and performance within virtual environments for patients with stroke. In Proceeding of 5th Intl Conf. Disability, Virtual Reality \& Assoc. Tech, 19-26.

[41] Klinger, E. 2005. Virtual reality therapy for social phobia: its efficacy through a control study. In Paper presented at Cybertherapy, Basal, Switzerland.

[42] Ko, H. 2000. Navigation in virtual haptic-only environments. In Proc. Workshop Guiding Users through Interactive Experiences: Usability Centred Design and Evaluation of Virtual 3D Environments, V. Paelke and S. Volbracht, eds, 123-134.

[43] Krishna, S.; Bala, S.; McDaniel, T. L.; McGuire, S.; and Panchanathan, S. 2010. Vibroglove: an assistive technology aid for conveying facial expressions. In Proc of CHI Extended Abstracts, 3637-3642.

[44] Lahav, O., and Mioduser, D. 2000. Multisensory virtual environment for supporting blind persons' acquisition of spatial cognitive mapping, orientation, and mobility skills. In Proc. 3rd Intl Conf. Disability, Virtual Reality \& Assoc. Tech. (ICDVRAT), Alghero, Italy, 53-58.

[45] Lecuyer, A.; Mobuchon, P.; Megard, C.; Perret, J.; Andriot, C.; and Colinot, J. P. 2003. Homere: a multimodal system for visually impaired people to explore virtual environments. In Proceeding of IEEE Virtual Reality ( VR03), 251-258.

[46] Loomis, J. 1992. Distal attribution and presence. Presence: Teleoperators and Virtual Environments 1(1):113119.

[47] Loureiro, R.; Harwin, W.; Nagai, K.; and Johnson, M. 2011. Advances in upper limb stroke rehabilitation: a technology push. Medical \& Biological Engineering \& Computing 49(10):1103-1118.

[48] Lum, P. S.; Burgar, C. G.; Van der Loos, M.; Shor, P. C.; Majmundar, M.; and Yap, R. 2005. The mime robotic system for upper-limb neuro-rehabilitation: results from a clinical trial in subacute stroke. In Proceedings of the 9th International Conference on Rehabilitation Robotics, 511-514.

[49] Macedonio, M.; Parsons, T.; Wiederhold, B.; and Rizzo, A. A. 2007. Immersiveness and physiological arousal within panoramic video-based virtual reality. $C y$ berPsychology and Behavior 10(4):508-515.

[50] Matsumoto, D., and Willingham, B. 2009. Spontaneous facial expressions of emotion of congenitally and noncongenitally blind individuals. Journal of Personality and Social Psychology 96(1):1-10. 
[51] McCall, C.; Blascovich, J.; Young, A.; and Persky, S. 2009. Proxemic behaviors as predictors of aggression towards black (but not white) males in an immersive virtual environment. Social Influence 4:138-154.

[52] McGookin, D.; Robertson, E.; and Brewster, S. 2010. Clutching at straws: using tangible interaction to provide non-visual access to graphs. In Proceedings of CHI 2010: Sound and Speech, 1715-1724.

[53] Meehan, M.; Insko, B.; Whitton, M.; and Brooks Jr., F. P. 2002. Physiological measures of presence in stressful virtual environments. ACM Transactions on Graphics (TOG) 21(3):645-652.

[54] Mohler, B.; Campos, J.; Weyel, M.; and Bülthoff, H. 2007. Gait parameters while walking in a head-mounted display virtual environment and the real world. In Proceedings of 13th Eurographics Symposium on Virtual Environments and 10th Immersive Projection Technology Workshop (IPT-EGVE 2007), 1-4.

[55] Moustakas, K.; Nikolakis, G.; Kostopoulos, K.; Tzovaras, D.; and Strintzis, M. G. 2007. Haptic rendering of visual data for the visually impaired. IEEE MultiMedia 14(1):62-72.

[56] Naruse, H.; Fujisawa, T. X.; Yatsuga, C.; Kubota, M.; Matsuo, H.; and et al. 2017. Increased anterior pelvic angle characterizes the gait of children with attention deficit/hyperactivity disorder (adhd). PLoSOne 12(1):113.

[57] Nef, T.; Mihelj, M.; and Riener, R. 2007. Armin: A robot for patient-cooperative arm therapy. Medical \& Biological Engineering \& Computing 45(9):887-900.

[58] Nikolakis, G.; Tzovaras, D.; Moustakidis, S.; and Strintzis, M. G. 2004. Cybergrasp and phantom integration: enhanced haptic access for visually impaired users. In Proc of the 9th International Conference Speech and Computer (SPECOM'2004), 507-513.

[59] Panëels, S. A., and Roberts, J. C. 2009. Haptic interaction techniques for exploring chart data. In Proceedings of the 4th International Conference on Haptic and Audio Interaction Design (HAID09), 31-40.

[60] Panëels, S. A.; Ritsos, P. D.; Rodgers, P. J.; and Roberts, J. C. 2013. Prototyping 3d haptic data visualizations. Computers \& Graphics 37(3):179-192.

[61] Parente, P., and Bishop, G. 2003. Bats: the blind audio tactile mapping system. In Proceedings of ACM Southeastern Conf. (ACMSE 03).

[62] Parsons, S.; Leonard, A.; and Mitchell, P. 2006. Virtual environments for social skills training: comments from two adolescents with autistic spectrum disorder. Computers \& Education 47(2):186-206.

[63] Pertaub, D. P.; Slater, M.; and Barker, C. 2002. An experiment on public speaking anxiety in response to three different types of virtual audience. Presence 11(1):6878.

[64] Phillips, L.; Ries, B.; Kaeding, M.; and Interrante, V. 2010. Gait parameters in stressful virtual environments.
In Proceedings of the 2nd IEEE VR 2010 Workshop on Perceptual Illusions in Virtual Environment, 16-18.

[65] Pistori, H. 2006. Computer vision and digital inclusion of persons with special needs: overview and state of art. In Proceedings of Computational modelling of objects represented in images fundamentals, methods and applications (CompImage 2006), 1-6.

[66] Popescu, V.; Burdea, G.; and Bouzit, M. 1999. Virtual reality simulation modeling for a haptic glove. In Proceedings Computer Animation (CA '99), 195-200.

[67] Rábago, C. A., and Wilken, J. M. 2011. Application of a mild traumatic brain injury rehabilitation program in a virtual realty environment: a case study. Journal of neurologic physical therapy: JNPT 35(4):185-193.

[68] Rand, D.; Kizony, R.; Feintuch, U.; and Katz, N. 2006. Comparison of two vr platforms for rehabilitation: Video capture versus hmd. Presence: Teleoperators and Virtual Environments 14(2):147160.

[69] Rector, K.; Bennett, C. L.; and Kientz, J. A. 2013. Eyes-free yoga: an exergame using depth cameras for blind \& low vision exercise. In Proceedings of the 15th International ACM SIGACCESS Conference on Computers and Accessibility (ASSETS '13), 1-8.

[70] Reiner, M.; Halevy, G.; Hecht, D.; Furman, M.; and Vainsencher, D. 2004. Discrimination among three types of anomalies in a visual-haptic virtual environment. Presence 13(50):317-320.

[71] Rizzo, A. A.; Ghahremani, K.; Pryor, L.; ; and Gardner, S. 2003. Immersive 360-degree panoramic video environments. In Human-Computer Interaction: Theory and Practice (J Jacko and C Stephanidis, Eds.), Vol. 1. L.A. Erlbaum, New York, 1233-1237.

[72] Rizzo, A.; Lange, B.; Buckwalter, J. G.; Forbell, E.; Kim, J.; Sagae, K.; Williams, J.; Difede, J.; Rothbaum, B. O.; Reger, G.; Parsons, T.; and Kenny, P. 2010. Simcoach: an intelligent virtual human system for providing healthcare information and support. International Journal of Disability and Human Development 10(4):213221.

[73] Rizzo, A. A.; Lange, B.; Buckwalter, J. G.; Forbell, E.; Kim, J.; Sagae, K.; Williams, J.; Rothbaum, B. O.; Difede, J.; Reger, G.; Parsons, T.; and Kenny, P. 2011. An intelligent virtual human system for providing healthcare information and support. In Proceedings of Medicine Meets Virtual Reality (MMVR18), 503-509.

[74] Roberts, J. C.; Franklin, K. M.; and Cullinane, J. 2002. Virtual haptic exploratory visualization of line graphs and charts. In Proceedings of Electronic Imaging (EI 2002).

[75] Samaraweera, G.; Guo, R.; and Quarles, J. 2013. Latency and avatars in virtual environments and the effects on gait for persons with mobility impairments. In Proceedings of IEEE Symposium on $3 D$ User Interfaces (3DUI 2013), Orlando, FL, 23-30. 
[76] Schultze, U. 2010. Embodiment and presence in virtual worlds: a review. Journal of Information Technology 25(4):434449.

[77] Selzer, M.; Clarke, S.; Cohen, L.; Duncan, P.; and Gage, F. 2006. Textbook of Neural Repair and Rehabilitation: Volume 2, Medical Neurorehabilitation. Cambridge Uni. Press.

[78] Sheik-Nainar, M. A., and Kaber, D. B. 2007. The utility of a virtual reality locomotion interface for studying gait behavior. Human Factors: The Journal of the Human Factors and Ergonomics Society 49(4):696-709.

[79] Slater, M., and Usoh, M. 1993. The influence of a virtual body on presence in immersive virtual environments. In Proceedings of the 3rd Annual Conference on Virtual Reality.

[80] Sledd, A., and OMalley, M. K. 2006. Performance enhancement of a haptic arm exoskeleton. In Proceedings of 14th Symposium on Haptic Interfaces for Virtual Environment and Teleoperator Systems, 375-381.

[81] Sloot, L. H.; van der Krogt, M. M.; and Harlaar, J. 2014a. Effects of adding a virtual reality environment to different modes of treadmill walking. Gait and Posture 39:939-945.

[82] Sloot, L. H.; van der Krogt, M. M.; and Harlaar, J. 2014b. Self-paced versus fixed speed treadmill walking. Gait and Posture 39:478484.

[83] Spirduso, W. W., and Asplund, L. A. 1995. Physical activity and cognitive function in the elderly. Quest 47(3):395-410.

[84] Suárez, A. A.; Santamara, M.; and Claudio, E. 2013. Virtual reality: a tool for treating phobias of heights. In Proceedings of Eleventh LACCEI Latin American and Caribbean Conference for Engineering and Technology (LACCEI2013)Innovation in Engineering, Technology and Education for Competitiveness and Prosperity, Cancun, Mexico.

[85] Sveistrup, H.; McComas, J.; Thornton, M.; Marshall, S.; Finestone, H.; McCormick, A.; Babulic, K.; and Mayhew, A. 2003. Experimental studies of virtual reality-delivered compared to conventional exercise programs for rehabilitation. CyberPsychology and Behavior 6(3):245-249.

[86] Tarr, M. J., and Warren, W. H. 2002. Virtual reality in behavioral neuroscience and beyond. Nature Neuroscience 5:1089-1092.

[87] Tierney, N. W.; Crouch, J.; Garcia, H.; Walker, M.; Van Lunen, B.; DeLeo, G.; Maihafer, G.; and Ringleb, S. 2007. Virtual reality in gait rehabilitation. In MODSIM World 2007 Conference and Expo, Virginia beach, VA.

[88] Tonelo, C. 2013. Mime rehab. prezi presentation, (http://prezi.com/rjmijrs65esp/mime_rehab/).

[89] van der Krogt, M. M.; Sloot, L. H.; Buizer, A. I.; and Harlaar, J. 2015. Kinetic comparison of walking on a treadmill versus over ground in children with cerebral palsy. Journal of Biomechanics 48:3586-3592.
[90] Van Scoy, F. L.; Kawai, T.; Darrah, M.; and Rash, C. 2000. Haptic display of mathematical functions for teaching mathematics to students with vision disabilities: design and proof of concept. In Proceedings of of IEEE International Conference on Robotics and Biomimetics (ROBIO), 31-40.

[91] Wagner, S. R. 2014. Haptic and exoskeleton devices for neurorehabilitation of upper limb paralysis: a state of art and a night-landing task. In NMU Master's Theses. Paper 2.

[92] Wellner, M.; Thüring, T.; Smajic, E.; von Zitzewitz, J.; Duschau-Wicke, A.; and Riener, R. 2007. Obstacle crossing in a virtual environment with the rehabilitation gait robot lokomat. In Proceedings of Medicine Meets Virtual Reality 15 - in vivo, in vitro, in silico: Designing the Next in Medicine, 497-499.

[93] Whitehead, A.; Johnston, H.; Nixon, N.; and Welch, J. 2010. Exergame effectiveness: what the numbers can tell us. In Proceedings of the 5th ACM SIGGRAPH Symposium on Video Games (Sandbox10), 55-62.

[94] Wu, J.; Song, A.; and Zou, C. 2007. A novel haptic texture display based on image processing. In Proceedings of of IEEE International Conference on Robotics and Biomimetics (ROBIO), 1315-1320.

[95] Yasmin, S., and Panchanathan, S. 2016. Haptic mirror: a platform for active exploration of facial expressions and social interaction by individuals who are blind. In Proceeding of 15th ACM SIGGRAPH Conference on VirtualReality Continuum and Its Applications in Industry (VRCAI 2016), 319-329.

[96] Yavuzer, G.; Senel, A.; Atay, M. B.; and Stam, H. J. 2008. "playstation eyetoy game improves upper extremity-related motor functioning in subacute stroke: a randomized controlled clinical trial. European Journal of Physical Medicine and Rehabilitation 44(3):237-244.

[97] Yu, W., and Brewster, S. 2002. Comparing two haptic interfaces for multimodal graph rendering. In Proceedings of 10th Symposium on Haptic Interfaces for Virtual Environment and Teleoperator Systems, HAPTICS, 3-9.

[98] Yu, W.; Cheung, K.; and Brewster, S. 2002. Automatic online haptic graph construction. In Proceedings of Eurohaptics (EH 02), 128-133.

[99] Yu, W.; Ramloll, R.; and Brewster, S. 1999. Exploring computer-generated line graphs through virtual touch. In Proc. Intl Symp. Interactive 3D Graphics (SI3D99), 97102.

[100] Yu, W.; Ramloll, R.; and Brewster, S. 2000. Haptic graph for blind computer users. In Proceedings of the First International Workshop on Haptic HumanComputer Interaction, 41-51. 
Table 1: VEs and target disabilities

\begin{tabular}{|c|c|c|}
\hline \hline Category & Types of VE & Target disabilities \\
\hline \hline 1 & Visual feedback-based VEs & Mobility impairment and cognitive impairment \\
\hline \hline 2 & Haptic VEs & Mobility impairment, cognitive impairment, and visual impairment \\
\hline 3 & VEs enhanced with exercise equipment & Mobility impairment \\
\hline
\end{tabular}

Table 2: Rehabilitation with visual feedback-based VEs

\begin{tabular}{|c|c|c|c|c|}
\hline $\begin{array}{c}\text { Target } \\
\text { disability }\end{array}$ & $\begin{array}{c}\text { Types of visual } \\
\text { feedback-based } \\
\text { VE }\end{array}$ & Devices & Characteristics & Rehabilitation addressed \\
\hline \multirow{4}{*}{$\begin{array}{l}\text { Mobility } \\
\text { impairment }\end{array}$} & \multirow[t]{2}{*}{$\begin{array}{l}\text { VE without full } \\
\text { body skeletal } \\
\text { tracking }\end{array}$} & $\begin{array}{l}\text { Interaction/ capture devices: } \\
\text { - } \quad \text { Camera, } \\
\text { - } \quad \text { Mouse/ joystick, } \\
\text { - } \quad \text { Wii-mote, } \\
\text { - } \quad \text { Sensoglove, and }\end{array}$ & \multirow[t]{2}{*}{$\begin{array}{l}\text { Allows a limited amount of } \\
\text { movement of different limbs } \\
\text { or the whole body }\end{array}$} & \multirow[t]{2}{*}{$\begin{array}{l}\text { Rehabilitation of hands, wrists, } \\
\text { frozen shoulders, or postural } \\
\text { imbalance. }\end{array}$} \\
\hline & & $\begin{array}{l}\text { Head Mounted Display } \\
(\mathrm{HMD}) \text { is used for better } \\
\text { immersion }\end{array}$ & & \\
\hline & \multirow[t]{2}{*}{$\begin{array}{l}\text { VE with full body } \\
\text { skeletal tracking }\end{array}$} & $\begin{array}{l}\text { Microsoft Kinect, motion } \\
\text { capture systems (e.g.,Vicon, } \\
\text { Oxford). }\end{array}$ & \multirow[t]{2}{*}{$\begin{array}{l}\text { Full body movement with } \\
\text { skeletal tracking }\end{array}$} & \multirow[t]{2}{*}{$\begin{array}{lrr}\text { Gait rehabilitation } & \text { by } \\
\text { continuous tracking } & \text { and } \\
\text { adjustment. } & & \end{array}$} \\
\hline & & HMD for better immersion & & \\
\hline \multirow[t]{2}{*}{$\begin{array}{l}\text { Cognitive } \\
\text { impairment }\end{array}$} & \multirow[t]{2}{*}{$\begin{array}{l}\text { N/A (full body } \\
\text { skeletal tracking is } \\
\text { not an option) }\end{array}$} & $\begin{array}{l}\text { Interaction with mouse/ } \\
\text { joystick in a desktop-based } \\
\text { environment or touchpad } \\
\text { based interaction }\end{array}$ & \multirow{2}{*}{$\begin{array}{l}\text { Emphasizes mental exercise to } \\
\text { contribute to mental } \\
\text { soundness by promoting both } \\
\text { structural and neurochemical } \\
\text { changes in the central nervous } \\
\text { system }\end{array}$} & \multirow[t]{2}{*}{$\begin{array}{l}\text { Traumatic brain injury (TBI), } \\
\text { autism, phobia, or hallucination } \\
\text { desensitization, } \\
\text { different } \\
\text { disorders. }\end{array}$} \\
\hline & & HMD for better immersion & & \\
\hline
\end{tabular}


Table 3: Rehabilitation with haptic VEs

\begin{tabular}{|c|c|c|c|c|}
\hline Type of VE & $\begin{array}{l}\text { Target } \\
\text { Disability }\end{array}$ & Devices & Characteristics & $\begin{array}{l}\text { Rehabilitation } \\
\text { addressed }\end{array}$ \\
\hline \multirow{5}{*}{$\begin{array}{l}\text { Visuo-haptic/ } \\
\text { haptic }\end{array}$} & \multirow{3}{*}{$\begin{array}{l}\text { Mobility } \\
\text { Impairment }\end{array}$} & $\begin{array}{l}\text { End effectors: } \\
\text { 3D Systems' Touch, } \\
\text { Touch X devices, Novint } \\
\text { Falcon, and more }\end{array}$ & $\begin{array}{l}\text { be able to touch or grip virtual } \\
\text { objects with a limited amount } \\
\text { of movements of fingers and } \\
\text { wrists }\end{array}$ & $\begin{array}{l}\text { increases patients' } \\
\text { finger dexterity and grip } \\
\text { force, and enhances } \\
\text { endurance of wrists and } \\
\text { upper limbs }\end{array}$ \\
\hline & & $\begin{array}{l}\text { Exoskeletal devices } \\
\text { (Ungrounded): } \\
\text { Rutgers Master II-ND } \\
\text { (RMII), CyberGrasp, and } \\
\text { more }\end{array}$ & $\begin{array}{l}\text { be able to touch, grasp, hold, } \\
\text { and squeeze virtual objects }\end{array}$ & $\begin{array}{l}\text { addresses orthopedic } \\
\text { rehabilitation of wrists, } \\
\text { fingers, upper limbs, } \\
\text { and so on }\end{array}$ \\
\hline & & $\begin{array}{l}\text { Exoskeletal devices } \\
\text { (Grounded): } \\
\text { Hand Wrist Assisting } \\
\text { Robotic Device } \\
\text { (HWARD), ARMin, } \\
\text { MAHI Exo-II, Haptic } \\
\text { Interaction Robot } \\
\text { (HIRO) III, and so on. }\end{array}$ & $\begin{array}{l}\text { be able to exert a large and } \\
\text { varied amount of force with } \\
\text { larger movements of limbs; } \\
\text { provides an orthotic shell to } \\
\text { support the entire limb, or the } \\
\text { whole hand up to the shoulder }\end{array}$ & $\begin{array}{l}\text { helps gradual recovery } \\
\text { of motor movements; } \\
\text { records kinematic and } \\
\text { kinetic outcomes such } \\
\text { as position, velocity, } \\
\text { acceleration and force } \\
\text { for further clinical } \\
\text { diagnosis. }\end{array}$ \\
\hline & $\begin{array}{l}\text { Cognitive } \\
\text { Impairment }\end{array}$ & $\begin{array}{l}\text { End effectors: } \\
\text { (As described above) }\end{array}$ & As described above & $\begin{array}{l}\text { improves attention and } \\
\text { concentration }\end{array}$ \\
\hline & $\begin{array}{l}\text { Visual } \\
\text { Impairment }\end{array}$ & $\begin{array}{l}\text { End effectors: } \\
\text { (As described above) }\end{array}$ & As described above & $\begin{array}{l}\text { helps the blind to } \\
\text { explore size, shape, } \\
\text { texture of the virtual } \\
\text { objects }\end{array}$ \\
\hline
\end{tabular}

Table 4: Rehabilitation in VEs enhanced with exercise equipment

\begin{tabular}{|c|c|c|c|c|}
\hline Type of VE & Target Disability & Devices & Characteristics & $\begin{array}{l}\text { Rehabilitation } \\
\text { addressed }\end{array}$ \\
\hline $\begin{array}{l}\text { Visual/ } \\
\text { visuo- } \\
\text { haptic }\end{array}$ & $\begin{array}{l}\text { Mobility impair- } \\
\text { ment }\end{array}$ & $\begin{array}{l}\text { - A large screen for projecting } \\
\text { visual scene, } \\
\text { - a treadmill or in situ bike, } \\
\text { - can be haptically enhanced, } \\
\text { - use of HMD for better im- } \\
\text { mersion, } \\
\text { - use of motion capture system } \\
\text { for accurate posture determi- } \\
\text { nation. }\end{array}$ & $\begin{array}{l}\text { - continuous feedback on } \\
\text { gait in an engaging envi- } \\
\text { ronment, } \\
\text { - scope of adjustment of } \\
\text { difficulty levels, task } \\
\text { complexity, and body } \\
\text { weight support. }\end{array}$ & $\begin{array}{l}\text { helps to } \\
\text { improve } \\
\text { postural } \\
\text { imbalance }\end{array}$ \\
\hline
\end{tabular}


Figure 1: Interaction/ capture devices in visual feedback based VEs: (a) Wiimote, (b) (Left) CyberGloveII and (Right) CyberGloveIII (Courtesy: CyberGlove Systems), (c) Microsoft Kinect, (d) Vicon Motion Capture System with infrared camera and retroreflective markers (Naruse et al. 2017).

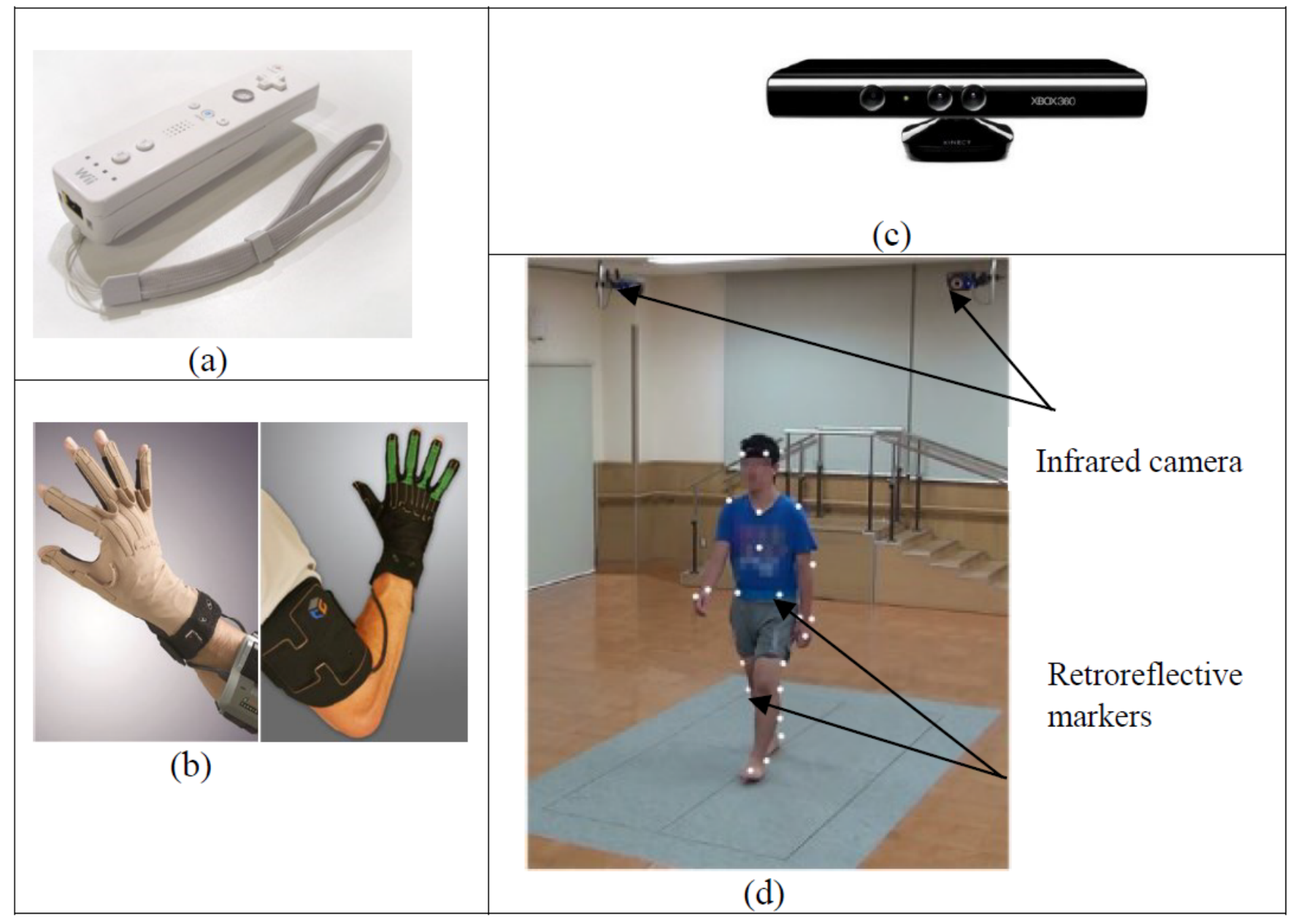


Figure 2: Different camera-based rehabilitation applications: (a) The rehabilitation gaming system (RGS) in use by a stroke patient, the patient wears data gloves for finger movements, and colored gloves are used to detect the movements of the hands (Cameiro et al. 2010), (b) a patient with spinal chord injury plays Birds and Balls using the Gesture Xtreme System (Kizony, Katz, and Weiss 2003), (c) a stroke patient plays Soccer using the Gesture Xtreme System (Kizony, Katz, and Weiss 2003), (d) Arrow Attack being played with bi-manual hand movements (Burke et al. 2009), (e) Mr. Chef being played in Playstation EyeToy (courtesy: Youtube), (f) using SIGUS platform, a virtual drum is being played with the movements of the patients face (Pistori 2006).

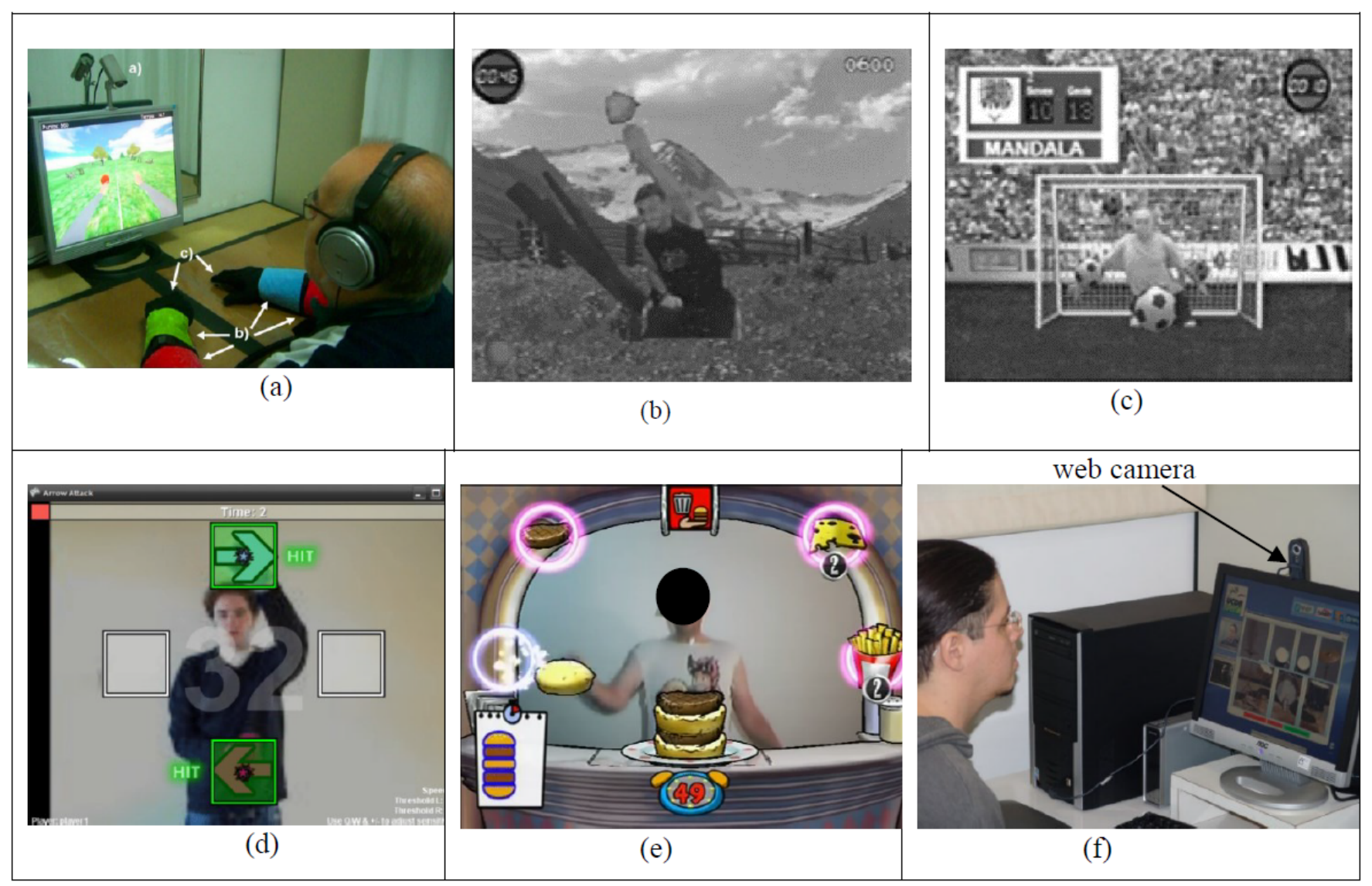


Figure 3: Wii-based rehabilitation: (a) a subjects different movements of hand while playing Helicopter with a Wiimote (strapped to his upper arm), (b) accurate muscle movement detection with an additional Wiimote: Wiimote at the lower arm detects elbow flexion and extension while the Wiimote on the upper arm cancels out compensation (Alankus et al. 2010).

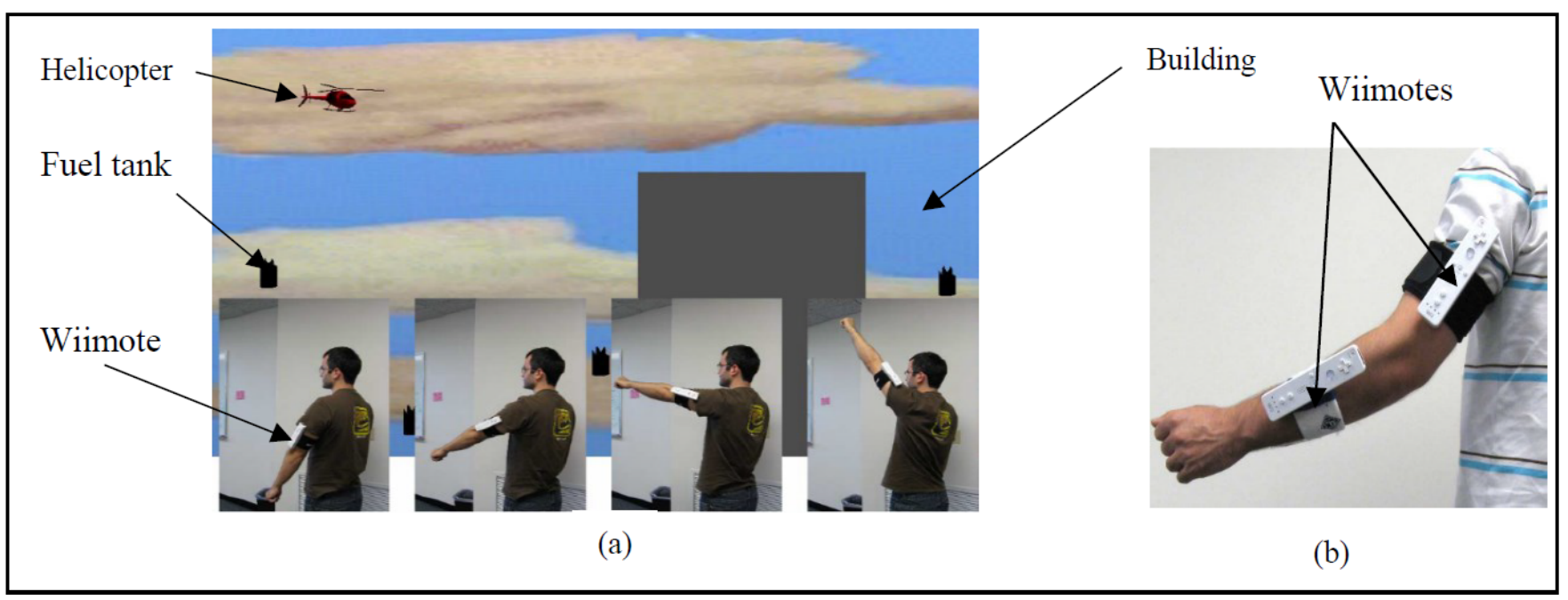

Figure 4: Simulated virtual environments for two sensor-based games: (a) Catch task and (b) Whack a mouse (Burke et al. 2009).

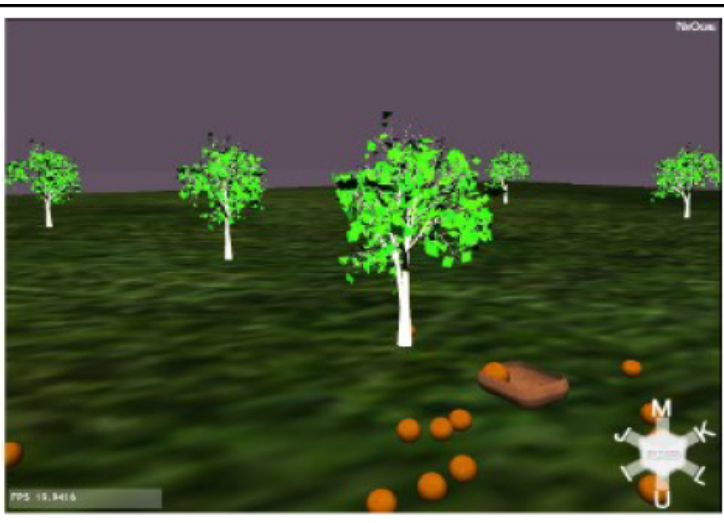

(a)

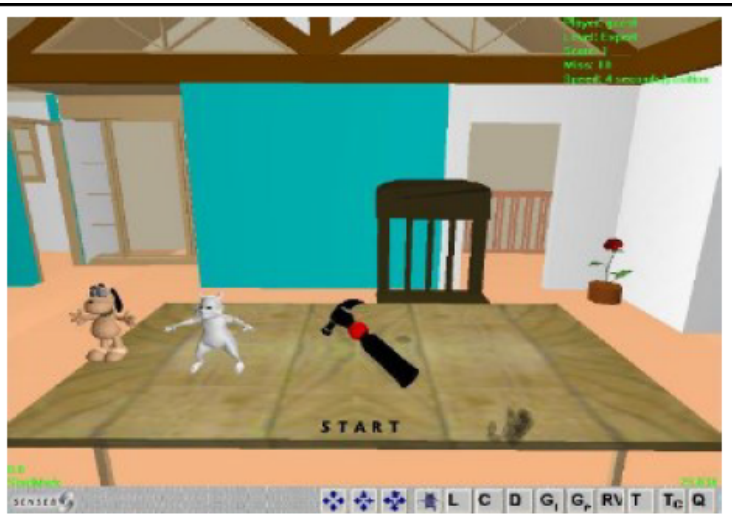

(b) 
Figure 5: Use of Microsoft Kinect in visual feedback-based rehab applications: (a) crouching and rising exercise and (b) half-crouching stability exercise with a car race game (Guo and Quarles 2013), (c) a user playing Game Cane through cane interactions and (d) the cane interface in detail in Game Cane (Espinoza, Cantu, and Quarles 2013), (e) in Eyes-free yoga, Warrior II yoga pose: subjects arms are at 45 degrees and need to be raised to equal to or greather than 80 degrees; the Kinect responds with a verbal correction, (f) skeletal tracking of a participants body movement in Eyes-free yoga (Rector, Bennett, and Kientz 2013).

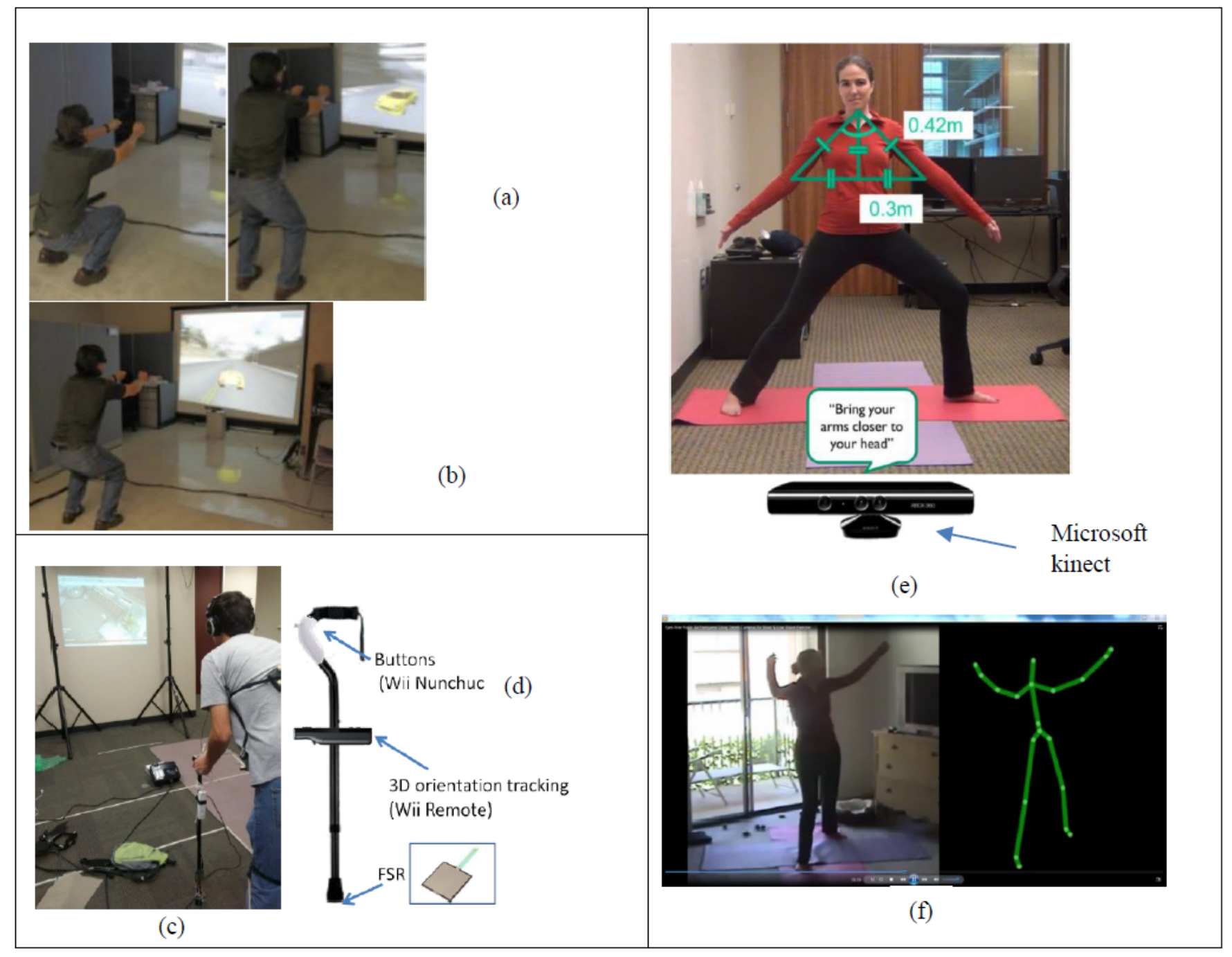


Figure 6: Virtual rehabilitation with motion capture systems : (a) visual cues in a VE to guide the patients about posture: a stick figure and a virtual human (Kiefer, Rhea, and Warren 2013), (b) (left) a student learning Tai chi wearing an HMD; (right) in a VE, the student as shown at the back is paired with his Tai chi instructor in front (Chua et al. 2003), (c) a subject wearing

an HMD with reflective markers on the body and the cane (Guo, Samaraweera, and Quarles 2013), (d) virtual avatars representing the subjects hands and the cane (Guo, Samaraweera, and Quarles 2013).

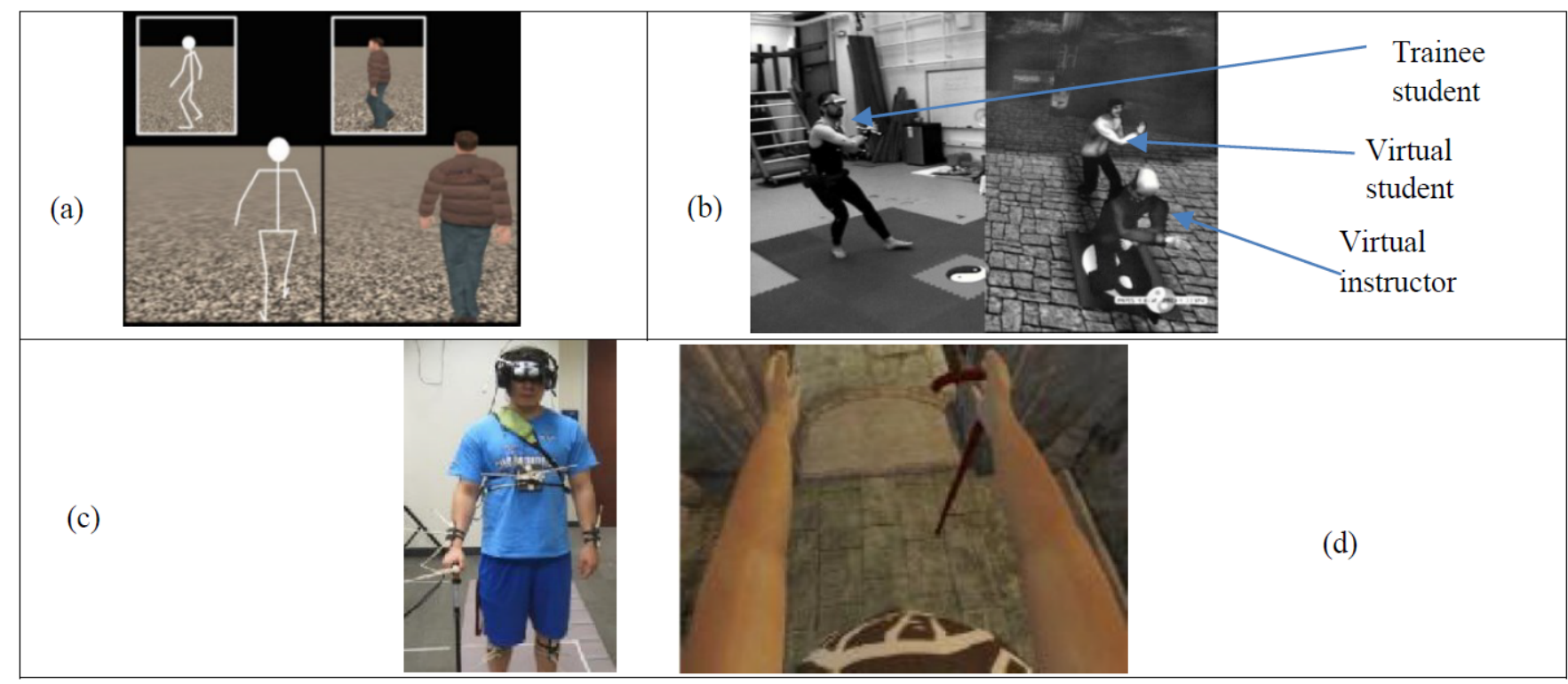

Figure 7: (a) A virtual kitchen to simulate a basic daily living skill: preparing a can of soup; (inset) a subjects mouse-based interaction with the simulated kitchen wearing an HMD (Christiansen et al. 1998), (b) a screenshot of a virtual caf illustrating the key functions of the program (Parsons, Leonard, and Mitchell 2006).

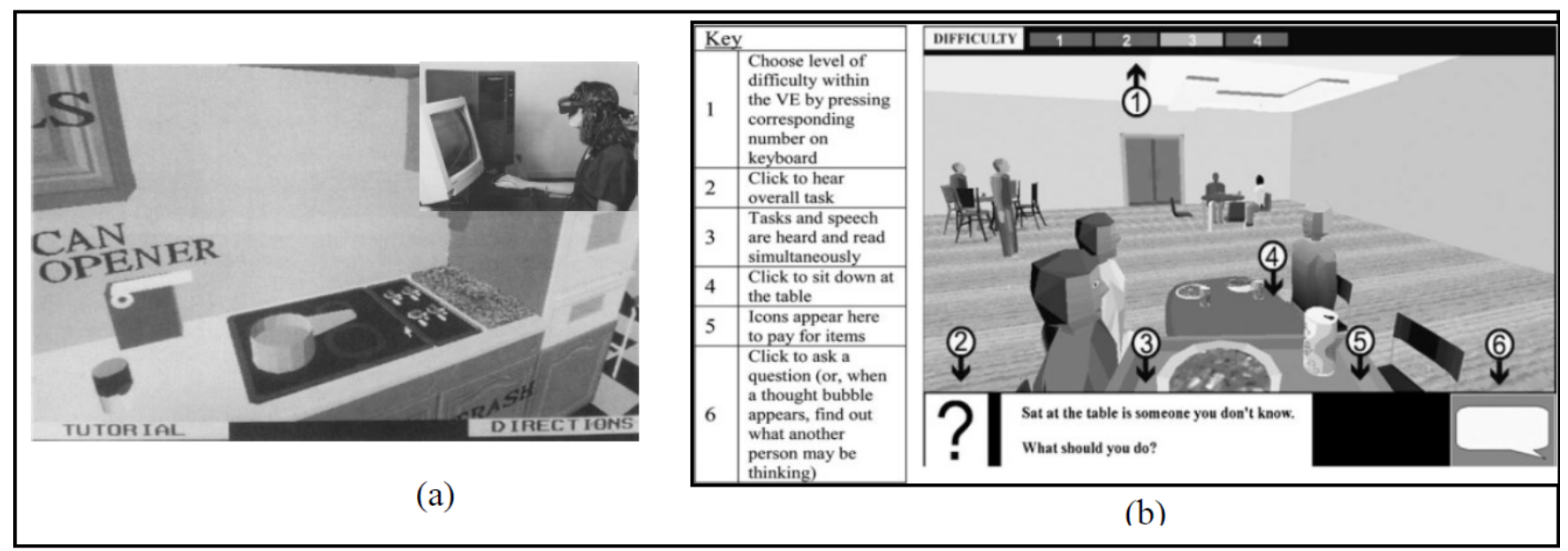

Figure 8: Some end effectors: (a) 3D Systems Touch, (b) 3D Systems Touch X, (c) Novint Technologies Novint Falcon.

(a) (b) (c)


Figure 9: Some popular exoskeleton devices: (a) RMII ND, (b) CyberGrasp, (c) HIRO III.

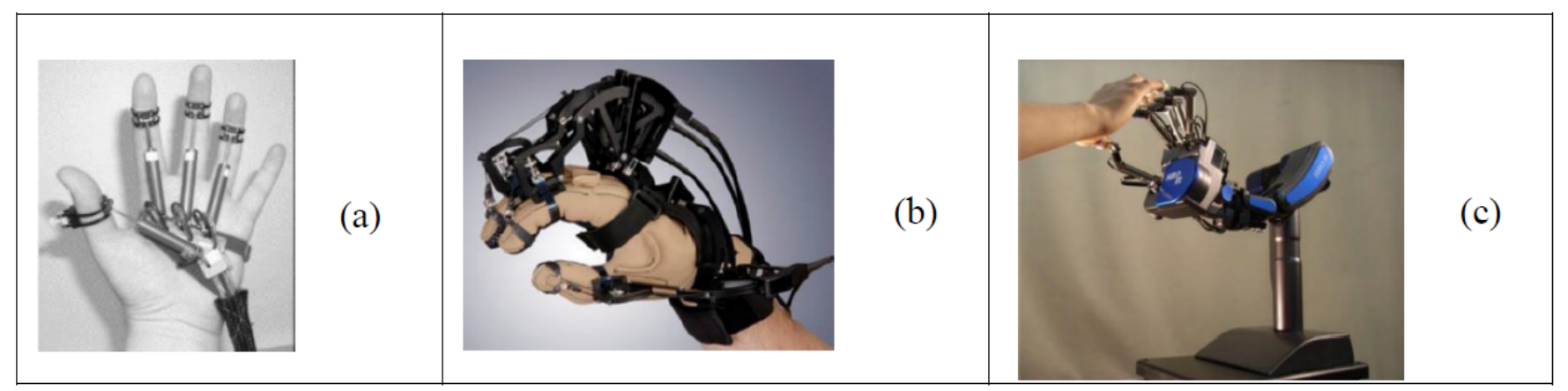

Figure 10: Rehabilitation with the end effectors: (a) A VR system with a desktop, a Touch X haptic device, and stereo glasses, (inset) a screenshot of the game Bricks and balls (Broeren, Rydmark, and Sunnerhagen 2004). (b) In VROOM system, a subject is interacting with a number of spheres with Phantom Premium 3.0 6 DOF device (Dvorkin et al. 2009).

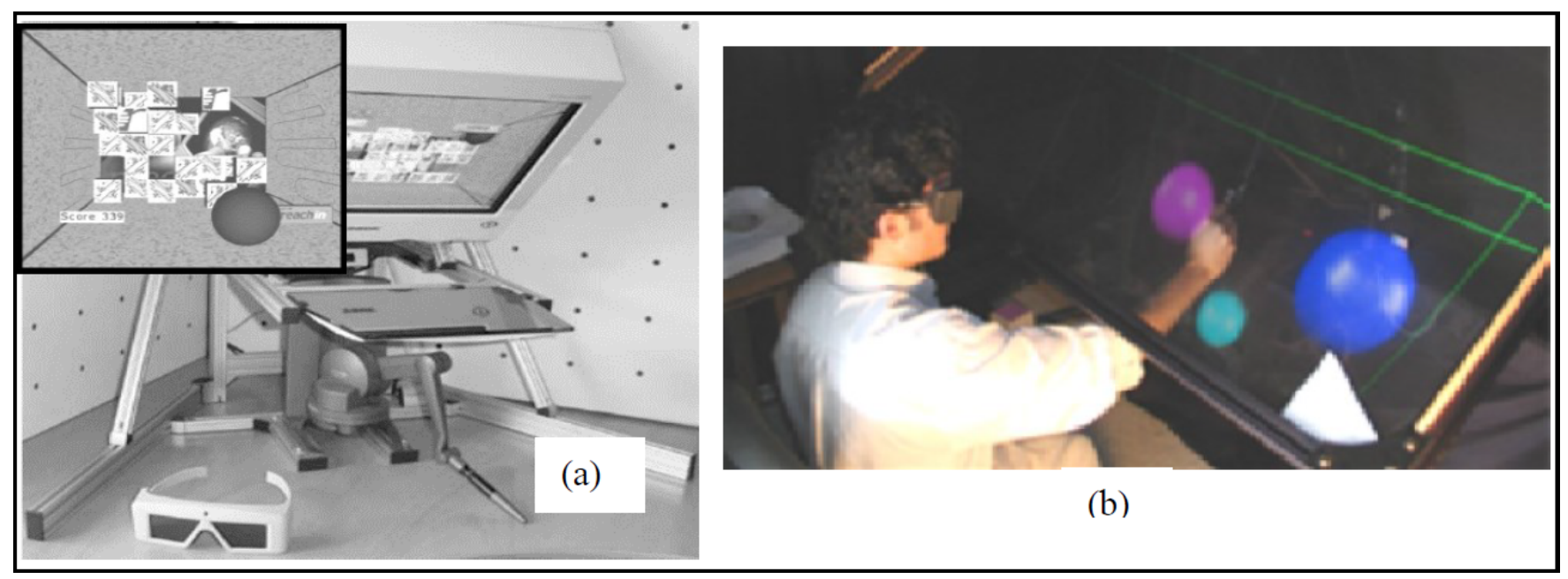

Figure 11: (Left) RM II worn by a subject (front view and back view of RMII). (Right) in a VE, the user is pressing a ball with RM II, corresponding force on each finger (except pinky finger) is displayed at the top (Heuser et al. 2007).

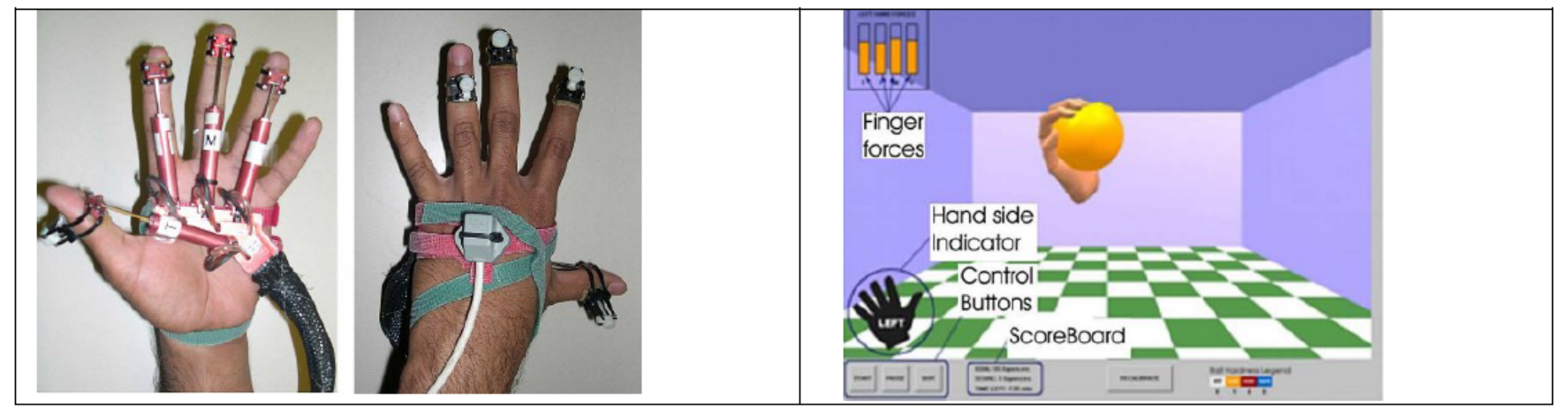


Figure 12: (a) HIRO III interface and a subjects interaction with a virtual object i.e., a cube. (b) Haptic fingertips (shown as red dots) while approaching an virtual object, (c) grasping the virtual object, and (d) lifting the virtual object (Endo et al. 2011).

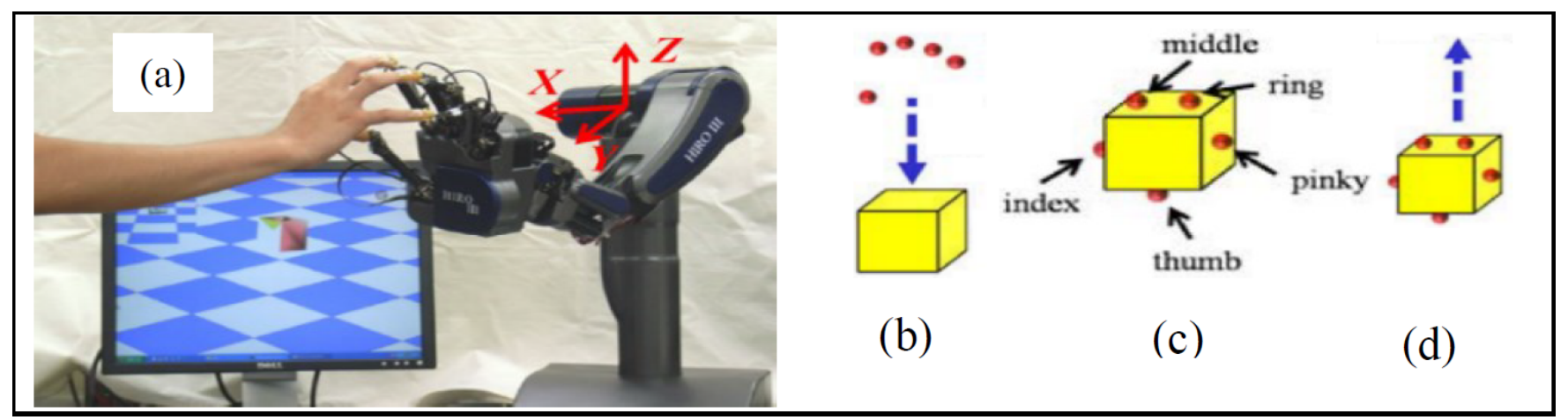

Figure 13: (a) A haptic line chart with V-shaped grooved lines, (inset) a blind individual is exploring the haptic line chart (Panëels et al. 2013), (b) in SoundBar Builder System, tangible V-shaped grooved bars are sonified (McGookin, Robertson, and Brewster 2010), (c) haptic pie chart (Yu and Brewster 2002), (d) haptic scatter plot with force model on the right (Panëels and Roberts 2009), (e) haptics-based representation of a brick surface with corresponding 3D height map on the right (Wu, Song, and Zou 2007).

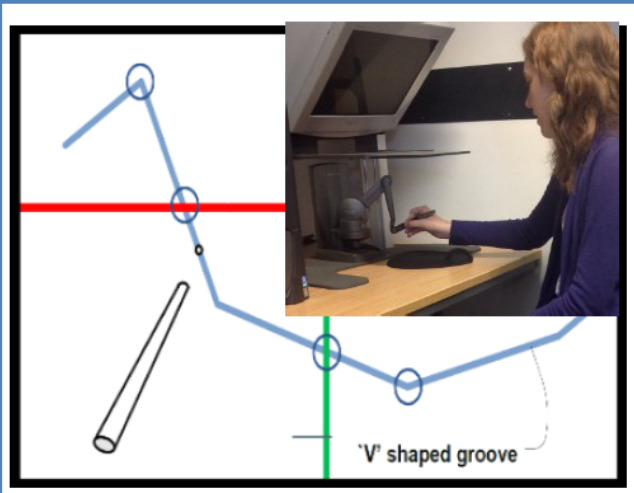

(a)

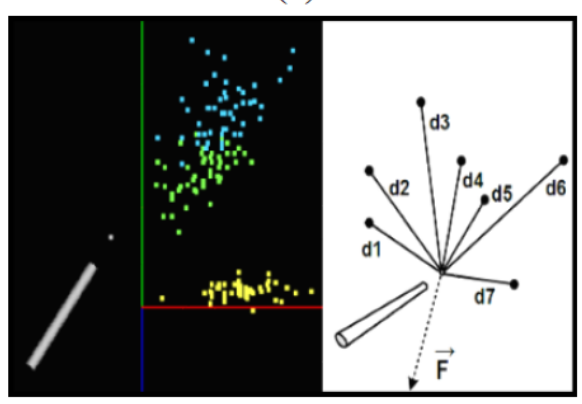

(d)

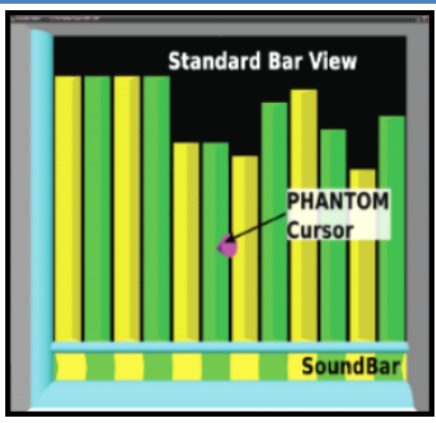

(b)

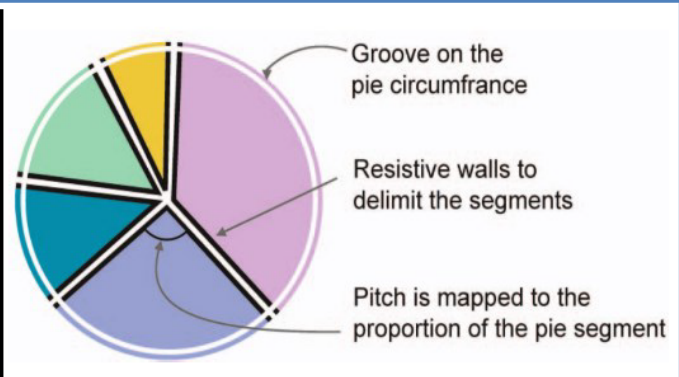

(c)

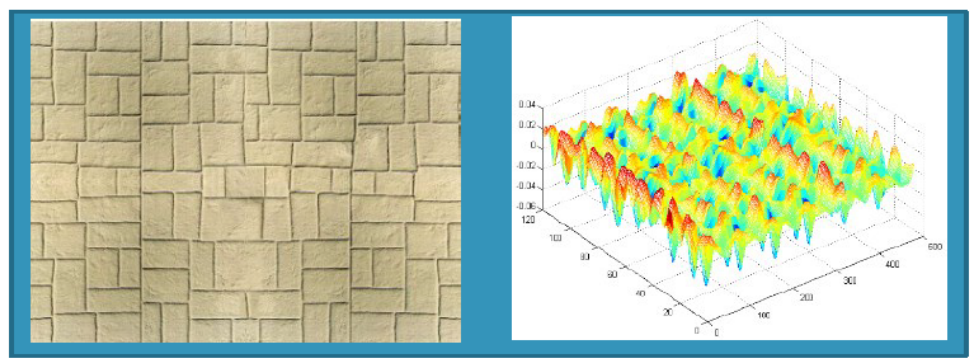

(e) 
Figure 14: (a) A blind individual is exploring the HOMERE system (Lecuyer et al. 2003), (b) Phantom desktop, CyberGlove, and CyberGrasp were combined to explore an object by selecting it from a category using audio-haptic menus (Nikolakis et al. 2004), (c) Haptic mirror in use by a blind individual (Yasmin and Panchanathan 2016).

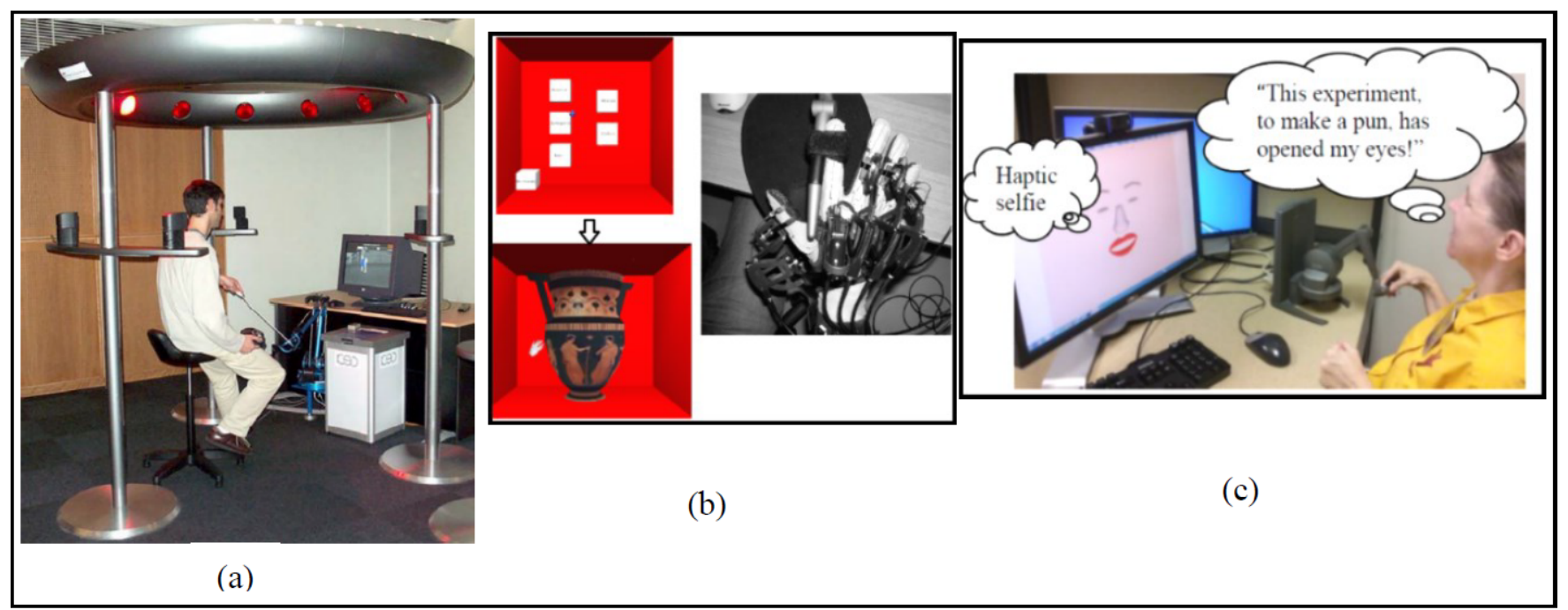

Figure 15: (a) The GRAIL experimental set-up consists of a dual-belt treadmill and 180 degrees field of view VR environment, (inset) different VR environments (Sloot, van der Krogt, and Harlaar 2014a). (b) In VR-gait system, a subject on a treadmill (connected to the overhead suspension device) with an inertial tracker attached to the hat, is looking at the VR environment on the monitor (Tierney et al. 2007).

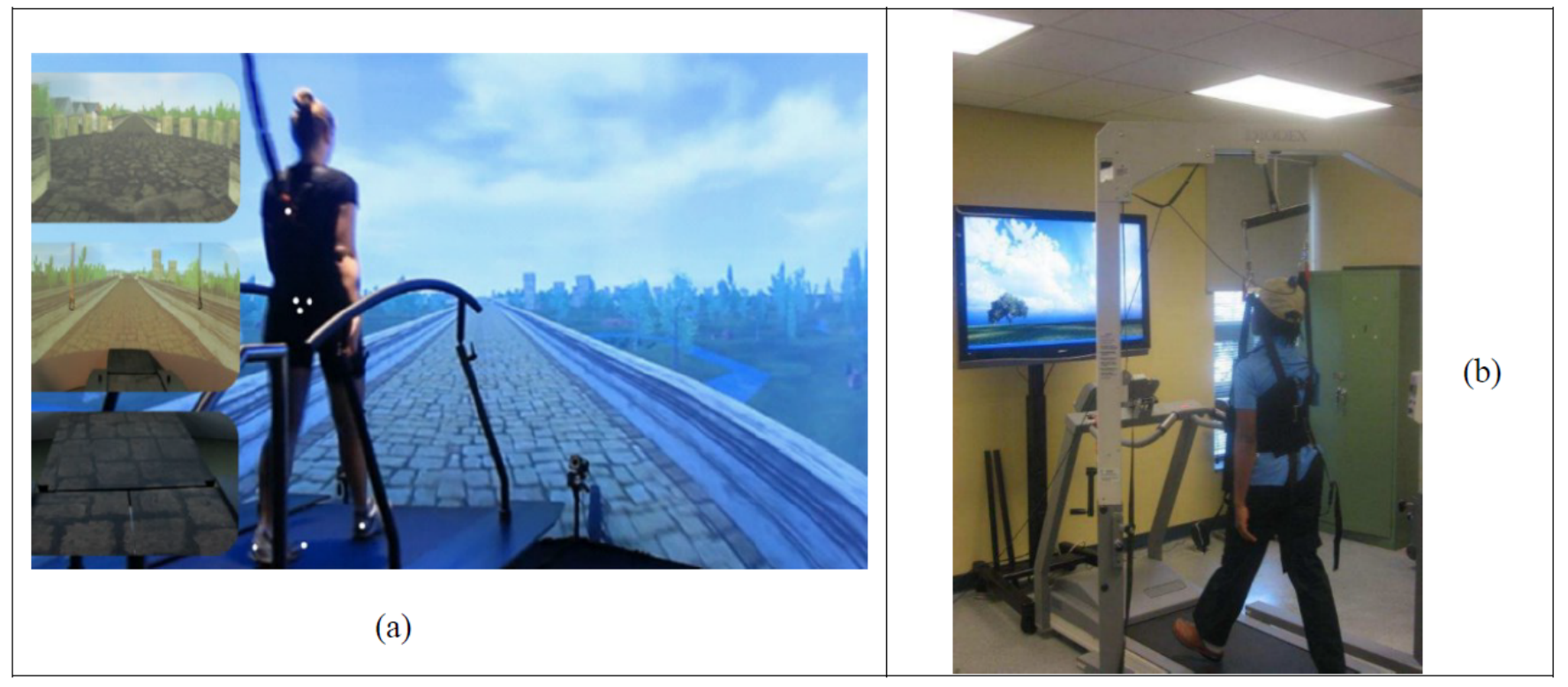


Figure 16: (a) A VR-enhanced bike system: (left) an overview of the bike system, (middle) the bike simulator, (right) the virtual road map (Kim, Song, and Kim 2001). (b) A VR-enhanced leg orthosis driven treadmill: (left) the rehabilitation robot locomat with leg orthosis, (right) a virtual avatar of the patient (Wellner et al. 2007).

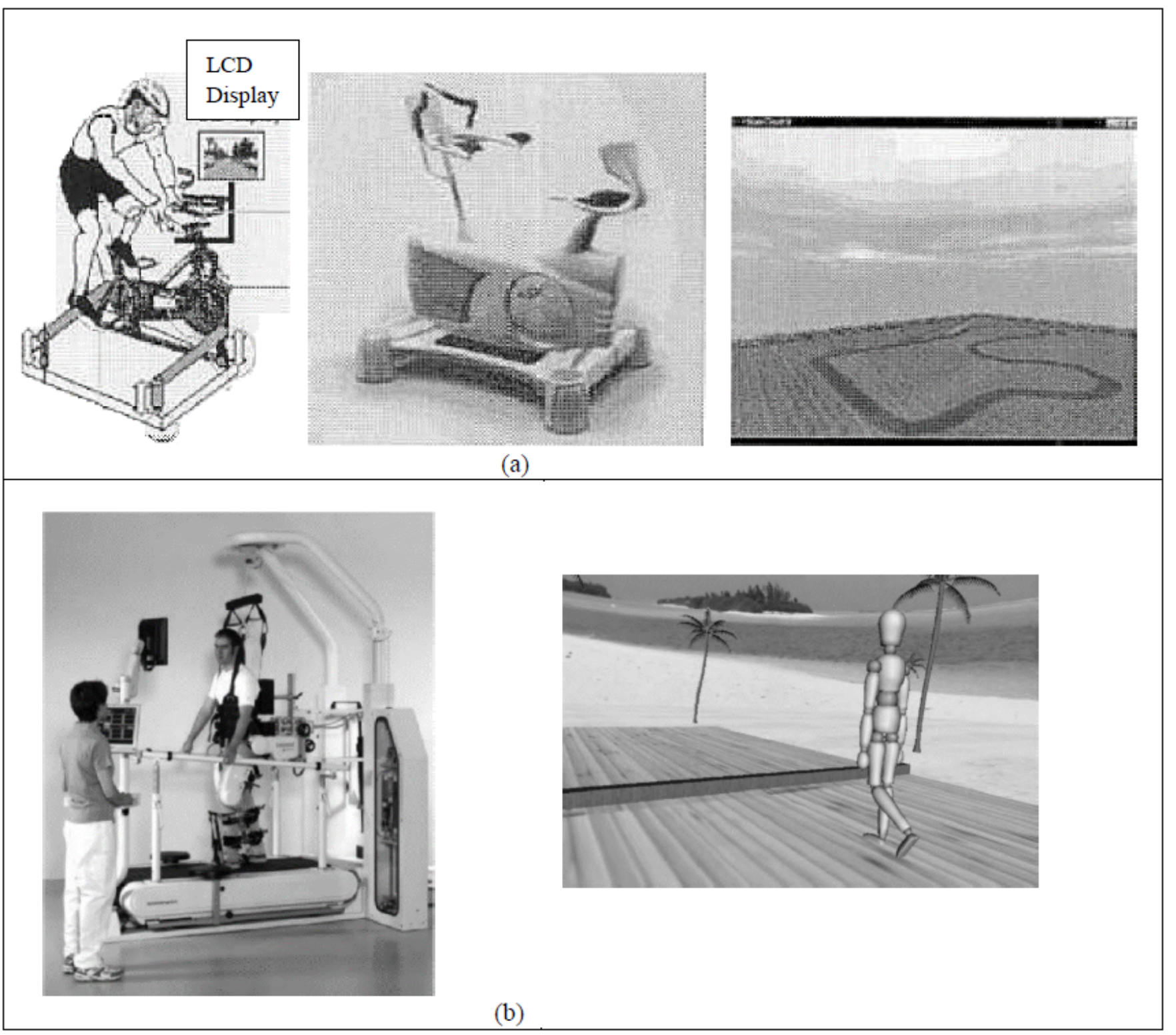


Figure 17: (a) A photograph by Hansi Durlach where two deafblind individuals (at the left and right) are communicating between themselves and with a sighted person (in the middle) using Tadoma technique and (b) how VR applications like haptic mirrors can facilitate communications among the deafblind individuals.

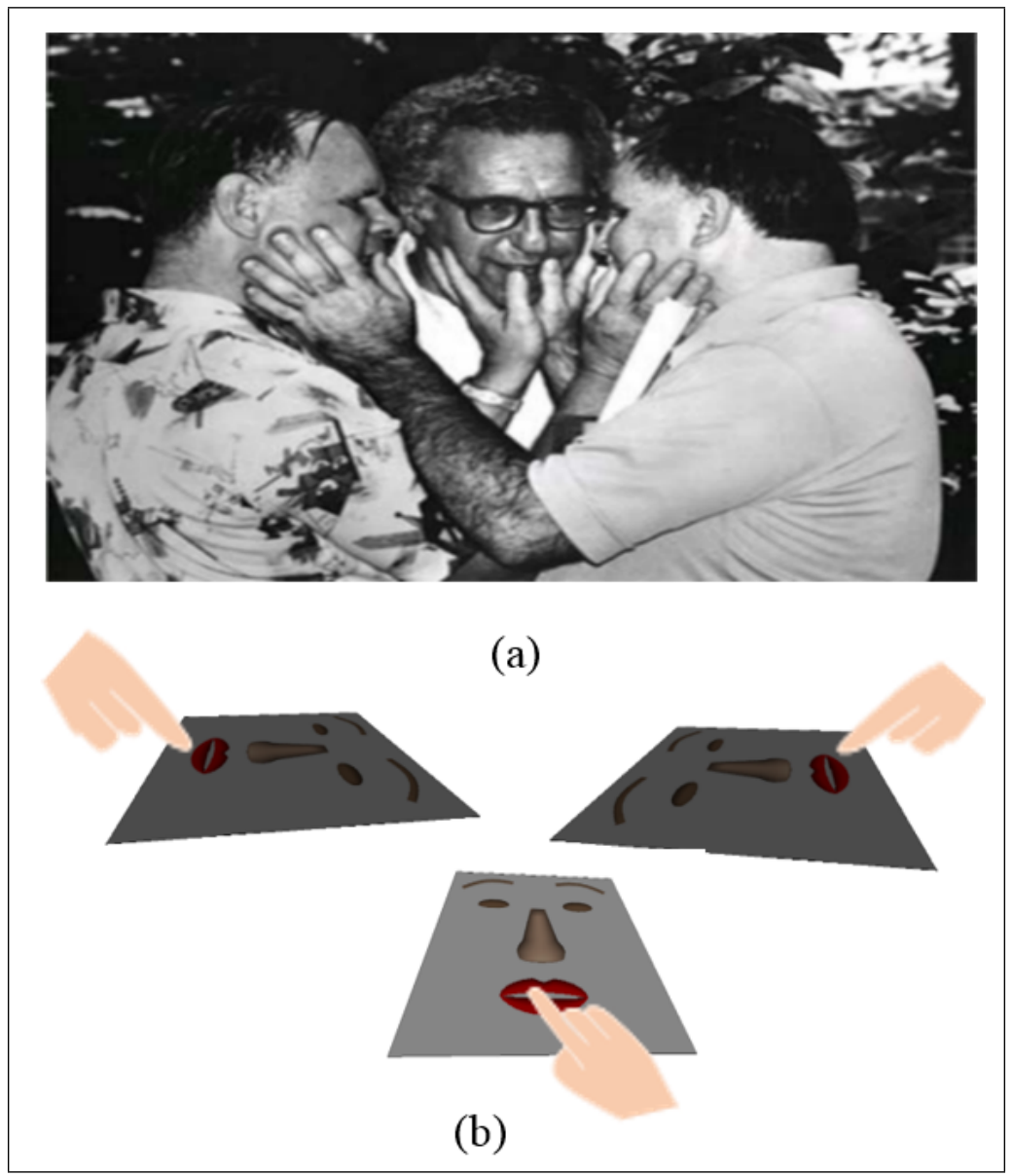

